\title{
Asphericity and clumpiness in the winds of Luminous Blue Variables ${ }^{\star}$
}

\author{
Ben Davies $^{1}$, René D. Oudmaijer ${ }^{1}$, and Jorick S. Vink ${ }^{2}$ \\ 1 School of Physics \& Astronomy, University of Leeds, Woodhouse Lane, Leeds LS2 9JT, UK \\ e-mail: [bd; roud]@ast. leeds.ac.uk \\ 2 Blackett Laboratory, Imperial College, Prince Consort Road, London SW7 2BZ, UK \\ e-mail: j.vink@imperial.ac.uk
}

Received 28 January 2005 / Accepted 8 May 2005

\begin{abstract}
We present the first systematic spectropolarimetric study of Luminous Blue Variables (LBVs) in the Galaxy and the Magellanic Clouds, in order to investigate the geometries of their winds. We find that at least half of our sample show changes in polarization across the strong $\mathrm{H} \alpha$ emission line, indicating that the light from the stars is intrinsically polarized and therefore that asphericity already exists at the base of the wind. Multi-epoch spectropolarimetry on four targets reveals variability in their intrinsic polarization. Three of these, AG Car, HR Car and P Cyg, show a position angle (PA) of polarization which appears random with time. Such behaviour can be explained by the presence of strong wind-inhomogeneities, or "clumps" within the wind. Only one star, R 127, shows variability at a constant PA, and hence evidence for axi-symmetry as well as clumpiness. However, if viewed at low inclination, and at limited temporal sampling, such a wind would produce a seemingly random polarization of the type observed in the other three stars. Time-resolved spectropolarimetric monitoring of LBVs is therefore required to determine if $\mathrm{LBV}$ winds are axi-symmetric in general.

The high fraction of LBVs ( $>50 \%$ ) showing intrinsic polarization is to be compared with the lower $\sim 20-25 \%$ for similar studies of their evolutionary neighbours, $\mathrm{O}$ supergiants and Wolf-Rayet stars. We anticipate that this higher incidence is due to the lower effective gravities of the LBVs, coupled with their variable temperatures within the bi-stability jump regime. This is also consistent with the higher incidence of wind asphericity that we find in LBVs with strong $\mathrm{H} \alpha$ emission and recent (last $\sim 10$ years) strong variability.
\end{abstract}

Key words. techniques: polarimetric - stars: mass-loss - stars: winds, outflows - stars: early-type - stars: activity stars: evolution

\section{Introduction}

Luminous Blue Variables (LBVs), or S Dor variables, are a class of evolved massive stars located close to the empirical upper-luminosity boundary on the H-R diagram, known as the Humphreys-Davidson (HD) limit (Humphreys \& Davidson 1994; van Genderen 2001, hereafter vG01). They exhibit photometric variability on three different scales: microvariations of a few tenths of a magnitude on timescales of weeks to months; variations of $\sim 1$ mag on timescales of years; and massive eruptions of $\gtrsim 2 \mathrm{mag}$, observed in only a handful of objects, $\eta$ Car and P Cyg being the most famous (see Lamers et al. 1998; Humphreys et al. 1999). At visual minimum their spectra resemble those of early-type supergiants with emission lines of $\mathrm{H} \mathrm{I}, \mathrm{He} \mathrm{I}$ and $\mathrm{Fe}$ II, with temperatures ranging from $15-30000 \mathrm{~K}$. At visual maximum, their temperatures are

* Data in Appendix A is available in electronic form at the CDS via anonymous ftp to cdsarc.u-strasbg.fr $(130.79 .128 .5)$ or via http://cdsweb.u-strasbg.fr/cgi-bin/qcat?]/A+A/439/1107 observed to fall to $\sim 9000 \mathrm{~K}$, as their radius increases and atmosphere cools (de Koter et al. 1996). Their mass-loss rates, of $\sim 10^{-5}-10^{-4} M_{\odot} \mathrm{yr}^{-1}$, vary with effective temperature, which can be understood by radiation pressure on spectral lines (Vink $\&$ de Koter 2002). The LBV phase itself is expected to last $\sim 10^{5} \mathrm{yr}$, and is thought to be a transitional phase experienced by stars with an initial mass $\gtrsim 25 M_{\odot}$ prior to the Wolf-Rayet stage (e.g. Maeder 1997).

With only a handful of exceptions, LBVs are surrounded by expanding aspherical nebulae (Weis 2003; Nota et al. 1995). These nebulae can be bipolar, elliptical or irregular; be discrete shells or clumpy, and are thought to result from the stars' episodic mass-loss phases. Nota et al. (1995) proposed that these nebulae may be formed by a spherically symmetric wind interacting with a pre-existing density contrast left over from a prior mass-loss episode, which was subsequently backed-up with models (Frank et al. 1995; Dwarkadas \& Balick 1998; Langer et al. 1999). It has however also been shown that such nebulae can be formed if the wind itself is axi-symmetric. 
Table 1. Log of the LBV observations. The signal-to-noise $(S / N)$ was calculated from the noise in flat regions of the continuum. The spectral resolution was calculated using the $F W H M$ of lines in the arc calibration spectra taken at the time and position of the observed star. All observations, including the archive data, were done at the AAT except P Cyg which was done at the WHT. All data was taken using a 1200R grating, except HD 160529 on 18/9/02 which was done using a 600V grating.

\begin{tabular}{|c|c|c|c|c|c|c|c|c|}
\hline \multirow[t]{2}{*}{ Name } & \multicolumn{2}{|c|}{ Coords (2000) } & \multirow[t]{2}{*}{$m_{\mathrm{V}}$} & \multirow[t]{2}{*}{ Obs. date } & \multirow{2}{*}{$\begin{array}{c}\text { Integration } \\
\text { time }(\mathrm{s})\end{array}$} & \multirow{2}{*}{$\begin{array}{c}\text { Slit } \\
\text { width (") }\end{array}$} & \multirow[t]{2}{*}{$S / N$} & \multirow{2}{*}{$\begin{array}{l}\text { Res } \\
(\AA)\end{array}$} \\
\hline & $\alpha$ & $\delta$ & & & & & & \\
\hline \multicolumn{9}{|l|}{ Galactic } \\
\hline AG Car & 105611.58 & -602712.8 & $8.1-6.0$ & 06.05 .94 & 960 & 1.0 & 600 & 1.5 \\
\hline & $"$ & $"$ & $"$ & 10.02 .03 & 600 & 1.0 & 500 & 1.1 \\
\hline HR Car & 102253.84 & -593728.4 & $8.7-7.4$ & 16.03 .92 & 3200 & 1.0 & 1300 & 1.5 \\
\hline$"$ & $"$ & $"$ & $"$ & 10.02 .03 & 680 & 1.0 & 600 & 1.1 \\
\hline$\eta \mathrm{Car}$ & 104503.59 & -594104.3 & 6.2 & 13.02 .03 & 12 & 1.0 & 300 & 1.1 \\
\hline Hen 3-519 & 105359.66 & -602644.3 & 11.0 & 06.05 .94 & 1600 & 1.0 & 500 & 1.5 \\
\hline$"$ & $"$ & $"$ & $"$ & 10.02 .03 & 8880 & 1.0 & 800 & 1.1 \\
\hline WRA 751 & 110840.4 & -604251 & $12.3-10.3$ & 11.02 .03 & 8400 & 1.5 & 300 & 1.3 \\
\hline P Cyg & 201747.2 & +380158.5 & 4.9 & 10.12 .03 & 64 & 1.0 & 700 & 0.8 \\
\hline$"$ & $"$ & $"$ & $"$ & 13.12 .03 & 48 & 1.0 & 600 & 0.8 \\
\hline HD 160529 & 174159.03 & -333013.7 & $6.95-6.4$ & 11.02 .03 & 620 & 1.0 & 450 & 1.1 \\
\hline \multicolumn{9}{|l|}{$S M C$} \\
\hline $\mathrm{R} 40$ & 010718.22 & -722803.7 & $10.7-9.9$ & 14.08 .03 & 8400 & 2.5 & 350 & 1.7 \\
\hline$"$ & $"$ & $"$ & $"$ & 15.08 .03 & 4400 & 2.0 & 300 & 1.5 \\
\hline \multicolumn{9}{|l|}{$L M C$} \\
\hline S Dor & 051814.3 & -691459 & $11.4-9.0$ & 11.02 .03 & 4800 & 1.0 & 500 & 1.1 \\
\hline R 71 & 050207.39 & -71 2013.1 & $11.0-9.9$ & 12.02 .03 & 7200 & 1.5 & 500 & 1.2 \\
\hline R 110 & 053051.48 & -690258.7 & $10.6-9.7$ & 12.02 .03 & 7200 & 1.5 & 500 & 1.3 \\
\hline R 116 & 053152.28 & -683238.9 & 10.6 & 13.02 .03 & 7200 & 1.0 & 600 & 1.1 \\
\hline R 127 & 053643.50 & -692945.0 & $11.4-8.8$ & 13.02 .03 & 2880 & 1.0 & 400 & 1.1 \\
\hline R 143 & 053851.60 & -690807.1 & $12.0-10.6$ & 14.02 .03 & 4800 & 2.0 & 350 & 1.5 \\
\hline
\end{tabular}

A bipolar wind can be created if the rotation rate is close to break-up and the star's flux is latitude dependent due to oblateness and gravity-darkening (e.g. Dwarkadas \& Owocki 2002). An equatorial wind may exist if the star is close to the bistability jump temperature where the recombination of Fe IV to Fe III below the sonic point of the wind leads to an increased opacity and a marked change in wind properties (Vink et al. 1999). Indeed, such a scenario has been suggested by Lamers $\&$ Pauldrach (1991) and Pelupessy et al. (2000) to explain the two-component wind model of the possibly related $\mathrm{B}[\mathrm{e}] \mathrm{su}-$ pergiants. However, direct observational investigations into the present-day wind geometries of evolved massive stars in general, and LBVs in particular, are thus far thin on the ground.

Evidence for aspherical stellar winds can be found through spectropolarimetry. Ionised circumstellar gas in the wind which has a flattened geometry on the sky, such as in a bi-polar or equatorially-enhanced flow, (electron) scatters continuum photons originating from the star. In the optically thin case, the scattered continuum light is then linearly polarized perpendicular to the plane of the flow. In the case of an optically thick wind, multiple scattering effects can mean the light is polarized parallel to the plane of the flow (Angel 1969; Wood et al. 1996). Emission-line radiation emanating from the ionized gas, which is formed over a much larger volume at a larger radius, undergoes less scattering and remains essentially unpolarized. Therefore a drop in polarization across an emission line is indicative of aspherical structures within the line-forming region. As the bulk of the polarization occurs within a couple of stellar radii where the circumstellar gas is most dense (Cassinelli et al. 1987), this signature tells us about the geometry at the very base of the wind.

Spectropolarimetric evidence for aspherical stellar winds exists for the Galactic LBVs AG Car (Leitherer et al. 1994; Schulte-Ladbeck et al. 1994), HR Car (Clampin et al. 1995), and P Cyg (Taylor et al. 1991; Nordsieck et al. 2001), as well as the Magellanic Cloud LBV R 127 (Schulte-Ladbeck et al. 1993). These studies deal only with individual objects, however, and until now no systematic study exists to determine whether LBVs in general undergo aspherical mass loss. Such studies do exist for $\mathrm{O}$ supergiants and Wolf-Rayet stars - of which LBVs are possibly the evolutionary mid-point - but evidence for significant intrinsic polarization was found in only $\sim 25 \%$ and $\sim 20 \%$ of these groups respectively (Harries et al. 2002, 1998). As LBVs are closer to their modified Eddington limit, have lower effective gravities, and are in the bi-stability jump regime, we may expect to find different mass-loss behaviour. In the first systematic spectropolarimetric study of LBVs, we will show that they do indeed show a higher proportion of intrinsic polarization, and hence that their wind geometries in general differ from those of their evolutionary neighbours. 
Table 2. Log of the observations of the stars in the spectral atlas. Columns show the same as Table 1, except Col. 4 which shows the MK spectral type of each of the stars. All observations were done at the AAT, and all used a 1200R grating with the exception of HD 183143 which used a $600 \mathrm{~V}$ grating. All stars were observed on 15/2/04 with the exception of HD 183143 and HD 32034 which were observed on 15/9/02 and $15 / 8 / 03$ respectively.

\begin{tabular}{lccccccc}
\hline \hline Name & \multicolumn{2}{c}{ Coords $(2000)$} & $\begin{array}{c}\text { Spec. } \\
\text { type }\end{array}$ & $\begin{array}{c}\text { Integration } \\
\text { time }(\mathrm{s})\end{array}$ & $\begin{array}{c}\text { Slit } \\
\text { width }\left({ }^{\prime \prime}\right)\end{array}$ & $\begin{array}{c}S / N \\
\text { ( }\end{array}$ & $\begin{array}{c}\text { Res. } \\
(\AA)\end{array}$ \\
\hline HR 5281 & 140625.2 & -594257 & B0Iae & 120 & 1.5 & 350 & 1.2 \\
HR 5027 & 132048.3 & -554803 & B0.5Iae & 590 & 1.5 & 550 & 1.2 \\
HR 6142 & 163141.8 & -414902 & B1Iae & 70 & 1.5 & 600 & 1.2 \\
HR 4806 & 123852.4 & -671135 & B1Ia & 1260 & 2 & 600 & 1.2 \\
HR 4653 & 121416.9 & -642431 & B1.5Iae & 530 & 2 & 700 & 1.2 \\
HR 4198 & 104240.6 & -591257 & B2.5Iae & 660 & 1 & 500 & 1.1 \\
HR 3940 & 095651.7 & -543404 & B5Ia & 100 & 2 & 1100 & 1.2 \\
HR 4611 & 120553.6 & -653249 & B6Iab & 600 & 1.5 & 450 & 1.2 \\
HD 183143 & 192726.6 & +181745 & B8Iae & 120 & 1 & 900 & 2.1 \\
HD 32034 & 045511.1 & -671011 & B9Iae & 960 & 2 & 100 & 1.2 \\
HR 4169 & 103727.1 & -584400 & A0Iae & 180 & 1.5 & 400 & 1.2 \\
HR 4228 & 104805.4 & -595509 & A0Ia & 200 & 1 & 400 & 1.1 \\
HR 3975 & 100720.0 & +164546 & A0Ia & 120 & 1 & 800 & 1.1 \\
HR 4352 & 114845.1 & -264459 & A6Ia & 120 & 1 & 700 & 1.1 \\
\hline
\end{tabular}

We begin in Sect. 2 by describing the observational technique and data-reduction steps. We describe in Sect. 3 an empirical method for estimating the stars' temperatures (and hence phase) at the epoch of observation, and displays the results of the spectropolarimetry. These results are discussed in Sect. 4.

\section{Observations and data reduction}

The linear spectropolarimetric data were taken during four observing runs. Three were done at the $3.9 \mathrm{~m}$ AAT using the $25 \mathrm{~cm}$ camera of the RGO Spectrograph in September 2002, February 2003 and August 2003. The fourth was done at the $4.2 \mathrm{~m}$ WHT using ISIS in December 2003. The spectroscopic data was taken on the AAT runs as part of bad-weather back-up programmes, and exactly the same set-up was used as the spectropolarimetry data, minus the polarization optics. The weather was mixed during each run, with some cloud around on some of the nights data was taken. The seeing varied from subarcsecond to $\gtrsim 2$ " conditions. Additionally, the AAT archive was searched for previous spectropolarimetric observations of LBVs. Only high/medium resolution, previously un-published data was selected.

The instrumental set-up was similar for all the AAT spectropolarimetry observations. A dekker mask with two holes of size $2.7^{\prime \prime}$ and separation $22.8^{\prime \prime}$ was placed in front of the slit in order to observe the target and the sky simultaneously. A half-wave plate is used to rotate the polarization of the incoming light, and a calcite block splits the light into two perpendicularly polarized beams (the $O$ and the $E$ rays), hence four spectra are recorded - the $O$ and $E$ beams, of both the target and the sky. One complete polarization observation consists of one exposure at four different waveplate positions $-0^{\circ}$ and $45^{\circ}$ (to measure Stokes $Q$ ), $22.5^{\circ}$ and $67.5^{\circ}$ (to measure Stokes $U$ ). Several sets of each object were obtained to check for repeatability of results, and also to avoid saturation of the CCD at the peak of the $\mathrm{H} \alpha$ line. Spectropolarimetric and zero-polarization standards were observed each night, and calibration spectra were taken after each target by observing a copper-argon lamp.

The August 2003 and February 2003 runs used a 1200R grating in conjunction with the EEV2 CCD windowed to $200 \times 2800$, giving a spectral range of $1250 \AA$ centred at $6500 \AA$. The September 2002 run used a $600 \mathrm{~V}$ grating in conjuction with the MITLL3 CCD windowed to $200 \times 3584$, giving a spectral range of $2690 \AA$ centred at $5600 \AA$. P Cygni was observed in December 2003 at the WHT, and the set-up is described in Vink et al. (2003). The MARCONI2 CCD was used in conjunction with the $1200 \mathrm{R}$ grating, which gave a range of $1055 \AA$ centred on $6500 \AA$ A. A log of the observations, including integration times and spectral resolution achieved is shown in Tables 1 and 2 .

The reduction steps included bias subtraction, flat-fielding, cosmic-ray removal, chip linearity correction, spectrum extraction and wavelength calibration. The sky spectra were subtracted from the object spectra in order to compensate for atmospheric features and extended nebulous emission which may contaminate the star aperture. These steps were done using the FIGARO package maintained by Starlink. Determination of the Stokes parameters was done using the Time-Series/Polarimetry (TSP) package, also maintained by Starlink. From the Stokes parameters, the degree of polarization $P$ and position-angle (PA) $\theta$ were found from,

$P=\sqrt{Q^{2}+U^{2}}$

$\theta=\frac{1}{2} \arctan \frac{U}{Q}$

PA calibration and wave-plate chromicity were corrected for using the Starlink package POLMAP, by fitting a 4th-degree polynomial to the spectra of polarized standards. The highfrequency "ripple" observed in high $S / N$ spectropolarimetry data, caused by internal reflections within the waveplate assembly (Aitken \& Hough 2001; Harries \& Howarth 1996), was corrected for using a Fourier transform algorithm, also within 
POLMAP. Instrumental polarization, determined from the zeropolarization standards, was found to be less than $0.1 \%$, with the origin in $Q-U$ space within the $1 \sigma$ rms scatter. As we are only interested in the internal precision of the instrument in order to detect changes in polarization across spectral features, this has not been corrected for.

The residuals between the measured Stokes parameters of polarized standards and their literature values showed a $1 \sigma$ scatter around zero of $\sim 0.2 \%$. The uncertainty in position-angle $\Delta \theta \sim 29^{\circ} \times \Delta P / P$, and is around $1.5^{\circ}$ for $P=4 \%$ and around $6^{\circ}$ for $P=1 \%$.

There were several extra steps required in the reduction. The $O$ and $E$ spectra, as well as spectra from different waveplate positions, were found to be spectrally misaligned with each other by a few tenths of a pixel. As the determination of the Stokes parameters essentially requires the spectra be divided by one another, this introduces artifacts in the polarization spectrum around sharp features. To compensate for this the spectra were cross-correlated with each other and realigned according to the measured shift.

Also, the $O$ and $E$ spectra have slightly different spectral resolutions, again by a few tenths of a pixel, despite efforts during focus set-up. We suspect that this is responsible for the erratic behaviour in the polarization spectrum in unresolved spectral features, such as a narrow P Cygni absorption components, which is highlighted by comparing the different sets of an object. Degrading the spectral resolution to make all spectra the same was not found to solve this problem. We suggest this is due to pixelation inherent in the data due to the finite separation of pixels on the CCD. We identified such artifacts by looking at the behaviour around narrow features in each data-set, and have either edited them out through linear interpolation or have been flagged in the text. This problem is unique to studies of objects with narrow features such as the discrete absorption components in LBV emission lines; it did not affect the results of other objects studied in previous papers (e.g. Oudmaijer \& Drew 1999; Vink et al. 2003).

\section{Results}

The spectra of all the stars show strong $\mathrm{H} \alpha$ emission, often with P Cygni absorption components. Many show emission lines of Fe II and [N II], whilst He I and Si II are seen in both emission and absorption. Of the objects for which more than one set exists, only AG Car exhibits significant spectral variability. The spectrum has changed from very reminiscent of HR Car to one with emission lines only, indicating a significant change in temperature and/or wind properties between the two epochs.

In Sect. 3.1 we estimate the temperature, and hence phase (whether at minimum or maximum) at the epoch of each observation. In Sect. 3.2 we display the results of the spectropolarimetry and discuss each object individually.

\subsection{Temperature and phase at observation}

The properties of line-driven stellar winds vary with effective temperature for two reasons - firstly, as a star cools a growing mismatch is formed between its flux-peak and the bulk of the driving lines in the UV (Lamers \& Cassinelli 1999). Secondly, at around $21000 \mathrm{~K}$ the opacity dramatically rises as the recombination of Fe IV to Fe III increases the number of opacity-enhancing ions (the so-called "bi-stability jump", see Vink et al. 1999). There is also evidence for a second bistability jump at $\sim 10000 \mathrm{~K}$, where Fe III recombines to Fe II (Lamers et al. 1995; Vink et al. 1999).

As LBV effective temperatures can vary from $9000 \mathrm{~K}$ to up to $30000 \mathrm{~K}$ on timescales of a few years, we may expect to find different wind properties at different epochs. If the star's temperature is latitude-dependent, due to e.g. rotation, we may expect to find differential wind properties between equator and pole as the star approaches its bi-stability jump, and therefore different wind geometries for the same star depending on the phase of the star at observation. This mechanism has been used to explain the two-component wind of $\mathrm{B}[\mathrm{e}]$ supergiants by Pelupessy et al. (2000). Here we estimate the temperature of the star at observation using two methods - comparing the photospheric spectral absorption features with an atlas of earlytype supergiants, and via the stars' bolometric correction from recent light-curves.

\subsubsection{A spectral atlas of early-type supergiants}

The spectra of emission line and non-emission line earlytype supergiants in the range 6240-6700 $\AA$ can be found in Appendix $\mathrm{A}^{1}$ Among the various spectral features, three lines in particular display a strong temperature dependence - the Si II $\lambda \lambda 6347,6371$ lines and the He I $\lambda 6678$ line, whilst no difference is observed between the emission and non-emission line stars. Figure 1 shows the measured equivalent widths $(E W \mathrm{~s})$ of these lines as a function of effective temperature. The $E W$ of the He I line clearly increases with increasing temperature, appearing at around $8000 \mathrm{~K}$ (spectral-type A7) and peaks at 19-21000 K (B1-B1.5), in agreement with SchmidtKaler (1982) who states that He I peaks at spectral type B0. The Si II lines decrease in $E W$ with temperature, peaking at 9-10000 K (B9-A2) and disappearing at around $19000 \mathrm{~K}$ (B2). This agrees well with Schmidt-Kaler (1982), who states that Si II lines peak at spectral-type AO $(9700 \mathrm{~K})$. We find no difference between emission and non-emission stars. It appears, therefore, that these three lines are reasonable indicators of effective stellar temperature in the range $9000 \mathrm{~K}-20000 \mathrm{~K}$ ( $\sim \mathrm{A} 2-\mathrm{B} 1)$. By comparing with the spectral features of the LBVs we can obtain an empirical estimate of $T_{\text {eff }}$, and therefore of the phase of the LBV at observation. This empirical relation will break down if the lines are in emission, but will only give a misleading temperature if there is a tiny amount of emission which only partially fills in the absorption.

\subsubsection{The bolometric correction method}

The light variations of LBVs occur at approximately constant bolometric luminosity (van Genderen 2001, VG01 hereafter). Their apparent variability is caused by their spectral energy

1 The full data for the spectral atlas is available in electronic form at the CDS. 


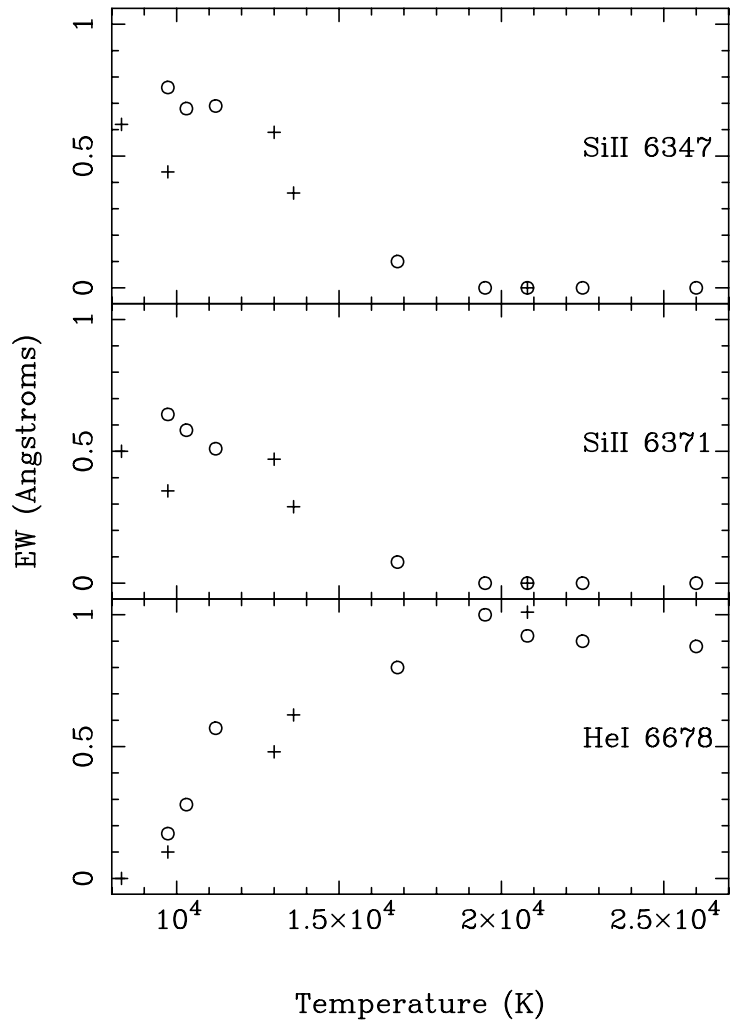

Fig. 1. The equivalent widths as a function of temperature for three photospheric absorption lines of early-type supergiants. Circles indicate stars with emission lines in their spectra, crosses stars with no emission. Note that the emission and non-emission stars follow the same relation. The uncertainties are of order the size of the plotting symbols and are not shown. The temperatures of the spectral types were obtained from Schmidt-Kaler (1982).

distribution (SED) shifting in and out of the visual band as their effective temperature changes. If we know the apparent bolometric magnitude, we can use the difference between this and the apparent magnitude at observation (the bolometric correction, $\mathrm{BC}$ ) to determine the star's temperature. This method has previously been used to determine LBV temperatures by Lamers et al. (1998).

The $\mathrm{BC}$ as a function of $T_{\text {eff }}$ for supergiants is quoted in Schmidt-Kaler (1982). We have fitted this to within 0.05 mags with the following relations:

$$
\begin{aligned}
& B C=+27.22-6.74 \log T_{\text {eff }},\left(\log T_{\text {eff }}>4.25\right) \\
& B C=+15.47-3.98 \log T_{\text {eff }},\left(\log T_{\text {eff }}<4.25\right) .
\end{aligned}
$$

VG01 quotes the $m_{\mathrm{V}}$ at visual minimum, with the associated $T_{\text {eff }}$, for each LBV as collated from multiple sources. From this, the BC and hence $m_{\mathrm{Bol}}$ can be calculated. The $m_{\mathrm{V}}$ at observation was obtained from the American Association of Variable Star Observers (AAVSO, http : //www . aavso.org/; Waagen 2005), and the BC at the epoch of observation was calculated from the difference between this and $m_{\mathrm{Bol}}$. Equations (3a) and ( $3 \mathrm{~b}$ ) were then used to calculate the $T_{\text {eff }}$ at observation.

The estimations of effective temperature at observation are shown in Table 3 . The two independent measures of temperature agree well for each object. Exceptions are marked in the
Table 3. Effective temperature at observation as calculated from the $\mathrm{BC}$ and spectral characteristics methods (see text for details). The two estimates agree well for most of the objects, exceptions are explained below.

\begin{tabular}{lcc}
\hline \hline Star & \multicolumn{2}{c}{$\log \left(T_{\text {eff }}\right)$} \\
& BC method & Abs. Line method \\
\hline AG Car (6/5/94) & $3.98 \pm 0.03$ & $4.00 \pm_{0.05}^{0.04}$ \\
AG Car (10/2/03) & $4.25 \pm 0.03$ & $-^{2}$ \\
HR Car (16/3/92) & $3.87 \pm 0.05$ & $3.98 \pm_{0.03}^{0.02}$ \\
HR Car (10/2/03) & $3.92 \pm 0.03$ & $3.95 \pm_{0.05}^{0.04}$ \\
7 Car & $-^{1}$ & $-{ }^{2}$ \\
Hen 3-519 & $-^{3}$ & $-^{2}$ \\
WRA 751 & $4.47 \pm 0.03$ & $4.40 . .4 .52$ \\
P Cyg & $-^{7}$ & $-^{2}$ \\
HD 160529 & $4.00 \pm 0.03$ & $3.95 \pm_{0.05}^{0.04}$ \\
R 40 & $4.04 \pm 0.08$ & $3.98 \pm_{0.04}^{0.03}$ \\
S Dor ${ }^{7}$ & $\gtrsim 3.93$ & $3.95 \pm_{0.04}^{0.03}$ \\
R 71 & $4.04 \pm 0.12$ & $4.19 \pm_{0.04}^{0.03}$ \\
R 110 & $4.04 \pm 0.08^{8}$ & $4.00 \pm_{0.03}^{0.02}$ \\
R 116 & $4.19 \pm 0.04$ & $\gtrsim 4.15^{6}$ \\
R 127 & $4.29 \pm 0.04$ & $4.13 \pm_{0.11}^{0.08}$ \\
R 143 & $-^{5}$ & $3.99 \pm_{0.05}^{0.04}$ \\
\hline
\end{tabular}

${ }^{1}$ The poor understanding of the intrinsic brightness of $\eta$ Car means that the BC method does not produce a reliable result.

${ }^{2}$ The star's spectrum does not contain the diagnostic absorption lines.

${ }^{3}$ An insufficient amount of data is available on the star's brightness history and brightness at the time of observation.

${ }^{4}$ There is disagreement on the star's temperature at minimum and maximum (as stated in vG01), giving two possible values of the star's temperature at observation using the $\mathrm{BC}$ method. Also the star's bolometric luminosity probably changes during outbursts (vG01), invalidating the BC method.

${ }^{5}$ No AAVSO data exists on this object.

${ }^{6}$ The Si II absorption lines appear to be filled in by emission in this object, so the temperature was determined from the He I line only.

${ }^{7} \mathrm{P}$ Cyg is not presently observed to be variable, therefore the $\mathrm{BC}$ method is not applicable.

${ }^{8} \mathrm{R} 110$ probably does not vary at constant $L_{\mathrm{BOL}}$, and so the $\mathrm{BC}$ method is invalid (vG01).

table, and probable explanations given in the footnotes. Figure 2 illustrates the phase of each LBV at observation by placing them on a HR diagram, along with their locations at visual minimum and maximum.

\subsection{Wind geometry}

As explained in Sect. 1, changes in polarization across emission lines supply evidence for aspherical stellar winds. The strength of the change in polarization is dependent on both the density contrast between different regions in the wind and the inclination angle. A non-detection could imply either the wind is symmetric about some axis (e.g. an equatorially-enhanced wind) but is oriented face-on; or the electron density at the base of 


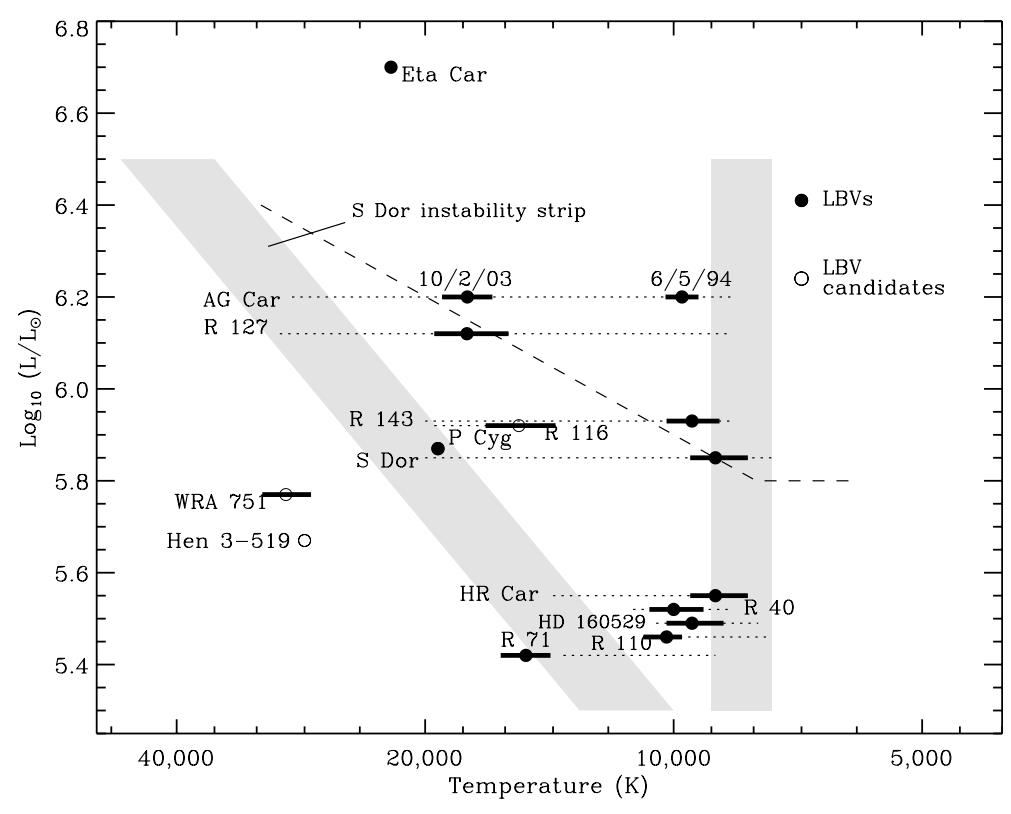

Fig. 2. HR diagram illustrating the temperature of each LBV at the epoch of observation. Filled and open circles show the temperature determined at observation for LBVs and candidate LBVs respectively, with the solid lines showing the error in each measurement. The luminosities, and minimum and maximum observed temperatures were collated from the literature by Smith et al. (2004). The Humphreys-Davidson limit is marked by a dashed line. $\eta$ Car, Hen 3-519 and P Cyg are marked on the plot despite the fact that their temperatures could not be determined - values are taken from Smith et al. and Cox et al. (1995). the wind is isotropic (i.e. the wind is spherically symmetric) to within the detection limit. This detection limit is dependent inversely on the $S / N$ of the spectrum, and the contrast of the emission line as the line-emission is "diluting" the polarized flux from the continuum. The detection limit per pixel for the maximum intrinsic polarization $\Delta P_{\text {int, max }}$ for each object is given by:

$\Delta P_{\text {int, } \max }(\%)=\frac{1}{S / N} \times \frac{L}{L-1} \times 100$

where $L$ is the line-to-continuum contrast. In the cases where no line-effect is observed, we state the systematic detection limit of our observations. Where line-effects are detected, the PA of the intrinsic component of the star's observed polarization is determined from the line-to-continuum vector in $Q-U$ space using Eqs. (1) and (2). Note that this is independent of the interstellar polarization (see below).

The continuum polarization is discussed below, and properties of the $\mathrm{H} \alpha$ emission as well as continuum polarization measurements are shown in Table 4. Object-by-object descriptions are listed below, starting with the Galactic objects. Of the 14 targets observed, 6 show definite line-effects, with one further possible detection. Of the 4 objects for which more than one observation exist, 3 show line-effects, and each of these three display polarimetric variability.

\subsubsection{Continuum polarization}

The observed polarization in the continuum is a superposition of multiple sources. We assume that this is broadly due to three mechanisms: scattering of light by free electrons at the base of the stellar wind; scattering by gas and dust in the star's nebula; and dichroic absorption by dust in the interstellar medium (ISM). Here we focus on the polarizing effects of asphericities in the stellar wind within a couple of stellar radii, which can vary on short timescales (days). For the purposes of this paper we will lump the nebula and the ISM together, and say the continuum polarization is due to the interstellar polarization (ISP) and electron scattering in the stellar wind.
Determination of the ISP, and hence separation of the two polarizing components is not straight-forward. The field-star method, attempted by Schulte-Ladbeck et al. (1994); Harries et al. (1998); Parthasarathy et al. (2000) amongst others, generally yields mixed results due to uncertain distances, the angular scale on which the ISP can change, and the unknown intrinsic polarization of the field stars. The line-centre method, used by Schulte-Ladbeck et al. (1993, 1994); Clampin et al. (1995); Oudmaijer et al. (1998), assumes that as the $\mathrm{H} \alpha$ lineemission is intrinsically unpolarized and formed over a much larger volume it will undergo negligible scattering and remain unpolarized. Therefore, if the line is much stronger than the continuum (such that the line contrast term in Eq. (4) tends to 1$)$, the $\mathrm{H} \alpha$ line-centre polarization is a measure of the ISP. Multiple Spectropolarimetric measurements of AG Car by Schulte-Ladbeck et al. (1994) showed that the polarization of $\mathrm{H} \alpha$ remained constant over $\sim 1.5$ years whilst the continuum polarization varied, showing that this method can be an effective method of determining the ISP.

\subsubsection{Object-by-object descriptions}

AG Car One of the prototype LBVs, AG Car has been highly variable for over 30 years with light variations of over 2 mag. It is surrounded by an elliptical nebula which has a hint of a bi-polar morphology, and a dynamical age of $\sim 10^{4}$ years (Nota et al. 1992). High-resolution HST images have shown that the nebula is made up of individual clumps, filaments and bubbles (Nota et al. 1995). Multiple spectropolarimetric observations revealed evidence for an axisymmetric stellar wind, with a PA alternating between perpendicular and parallel to the majoraxis of the nebula (Schulte-Ladbeck et al. 1994, SL94 hereafter). SL94 attributed this to either a wind which flips between an equatorial- and polar-enhanced wind, or multiple-scattering effects due to changes in optical depth.

The data from both epochs show line-effects (see Fig. 3). The archive data from 1994 had problems with varying 
Table 4. The observed $\mathrm{H} \alpha$ data. The first column denotes whether or not a line-effect was detected; the second column shows the contrast between the line-peak and the continuum to an accuracy of 0.1 ; the equivalent-widths and $F W H M$ are measured to an accuracy of $\sim 5 \%$. The continuum polarization was measured from featureless sections either side of the emission line. As the $\mathrm{R} 40$ data from consecutive nights showed no variability, the data was combined to improve the $S / N$. The quoted uncertainties do not include the external errors, which we estimate to be $0.1-0.2 \%$ (see Sect. 2).

\begin{tabular}{|c|c|c|c|c|c|c|}
\hline \multirow[t]{2}{*}{ Name } & \multirow{2}{*}{$\begin{array}{l}\text { Line- } \\
\text { effect? }\end{array}$} & \multirow{2}{*}{$\begin{array}{c}\text { Line-cont. } \\
\text { contrast }\end{array}$} & \multirow{2}{*}{$\begin{array}{l}E W \\
(\AA)\end{array}$} & \multirow{2}{*}{$\begin{array}{c}F W H M \\
\left(\mathrm{~km} \mathrm{~s}^{-1}\right)\end{array}$} & \multicolumn{2}{|c|}{ Cont. Pol. ( $R$-band) } \\
\hline & & & & & $P(\%)$ & $\theta\left(^{\circ}\right)$ \\
\hline \multicolumn{7}{|l|}{ Galactic } \\
\hline AG Car (6/5/94) & $\mathrm{Y}$ & 17.5 & -80 & 117 & $0.95 \pm 0.01$ & $160.1 \pm 0.2$ \\
\hline${ }_{(10 / 2 / 03)}$ & $\mathrm{Y}$ & 25 & -119 & 150 & $1.09 \pm 0.01$ & $132.6 \pm 0.4$ \\
\hline HR Car (16/3/92) & $\mathrm{Y}$ & 16 & -59 & 115 & $4.22 \pm 0.01$ & $129.7 \pm 0.1$ \\
\hline${ }_{(10 / 2 / 03)}$ & $\mathrm{Y}$ & 18 & -90 & 95 & $3.37 \pm 0.01$ & $133.9 \pm 0.1$ \\
\hline$\eta \mathrm{Car}$ & $\mathrm{Y}$ & 100 & -1480 & 450 & $5.71 \pm 0.04$ & $76.4 \pm 0.2$ \\
\hline Hen 3-519 (6/5/94) & $\mathrm{N}$ & 17 & -197 & 475 & $2.90 \pm 0.02$ & $97.6 \pm 0.2$ \\
\hline${ }_{(10 / 2 / 03)}$ & $\mathrm{Y}$ & 22 & -220 & 435 & $3.05 \pm 0.01$ & $99.2 \pm 0.1$ \\
\hline WRA 751 & $\mathrm{~N}$ & 4.2 & -10 & 90 & $2.29 \pm 0.01$ & $58.7 \pm 0.1$ \\
\hline P Cyg (10/12/03) & $\mathrm{N}$ & 18 & -63 & 167 & $0.99 \pm 0.02$ & $29.3 \pm 0.4$ \\
\hline$"_{(13 / 12 / 03)}$ & $\mathrm{N}$ & 18 & -64 & 170 & $0.93 \pm 0.02$ & $29.3 \pm 0.6$ \\
\hline HD 160529 & $\mathrm{~N}$ & 2 & -4.4 & 80 & $6.97 \pm 0.01$ & $21.2 \pm 0.1$ \\
\hline \multicolumn{7}{|l|}{$S M C$} \\
\hline $\mathrm{R} 40$ & $\mathrm{~N}$ & 4.6 & -15 & 87 & $0.28 \pm 0.03$ & $92.9 \pm 2.6$ \\
\hline \multicolumn{7}{|l|}{$L M C$} \\
\hline S Dor & $?$ & 8.3 & -34 & 100 & $0.24 \pm 0.02$ & $72.6 \pm 2.6$ \\
\hline R 71 & $\mathrm{~N}$ & 2.0 & -7.8 & 200 & $0.31 \pm 0.03$ & $60.1 \pm 2.3$ \\
\hline R 110 & $\mathrm{Y}$ & 26 & -96 & 100 & $1.50 \pm 0.03$ & $32.1 \pm 0.6$ \\
\hline R 116 & $\mathrm{~N}$ & 2.5 & -6.6 & 130 & $0.51 \pm 0.02$ & $28.6 \pm 1.0$ \\
\hline R 127 & $\mathrm{Y}$ & 40 & -114 & 78 & $0.50 \pm 0.01$ & $33.4 \pm 0.5$ \\
\hline R 143 & $\mathrm{~N}$ & 6.4 & -20 & 100 & $2.93 \pm 0.02$ & $73.3 \pm 0.2$ \\
\hline
\end{tabular}

spectral resolutions from set to set. Consequently, erratic polarization behaviour around the emission line was observed, and has been edited out in order to show the broad PA rotation coincident with the broad wings. Similar behaviour is seen in the 2003 data, but the continuum PA has changed by $\sim 30^{\circ}$. The varying PA of the observed polarization alone is evidence for an intrinsic component, as the interstellar polarization (ISP) will not vary on these timescales (see Sect. 3.2.1).

The line-centre polarization of AG Car has previously been shown to remain roughly constant, and is therefore probably a good estimate of the ISP (SL94, see Sect. 3.2.1). As shown in Fig. 3, the ISP measured by SL94 agrees well with the line-centre polarization of our 2003 data. The full $Q-U$ excursion of the 1994 data has been attenuated due to the linecentre being edited out, but it can clearly be seen to point towards the same region of $Q-U$ space. The mean of the line-centre polarizations, including those measured by SL94, is $Q=0.36 \pm 0.07 ; U=-1.08 \pm 0.09$.

Due to the attenuation of the 1994 data, the intrinsic polarization is measured from the line-wings, and is found to be $P>0.26 \pm 0.03 \%, \theta=26 \pm 3^{\circ}$. The intrinsic polarization of the 2003 data is $P=0.41 \pm 0.03 \%, \theta=84 \pm 2^{\circ}$. A weak line-effect is also seen across the He I $\lambda 6678$ line which is in emission at this epoch, with a similar PA to $\mathrm{H} \alpha$. However, line-effects are not observed across any other emission lines. The PAs from both epochs, $26^{\circ}$ and $84^{\circ}$, appear to be aligned with neither the nebula $\left(\sim 135^{\circ}\right)$, nor the jet $\left(\sim 35^{\circ}\right)$.

Looking at our data and the measurements of SL94, it can be seen that the intrinsic polarization of AG Car varies greatly from epoch to epoch (Fig. 3). The data from SL94 showed the intrinsic polarization vector flipping from one side of the origin in $Q-U$ space to the other, leading them to suggest that the intrinsic polarization varied between two preferred planes $90^{\circ}$ to each other. These planes aligned with the major and minor axes of the coronographic image of the AG Car nebula in Clampin et al. (1993). Our observations lie in neither of these planes, with the archive data being taken only $\sim 6$ months after the last SL94 observation. The continuum measurements of Leitherer et al. (1994, not shown in Fig. 3) are different again. If the wind was indeed flipping from an equatorial to a polar flow, we would expect the polarization of any intermediate phases to lie along the same axis in $Q-U$ space but with a smaller lineto-continuum vector. This is not observed here, with strong depolarizations observed at all epochs at unrelated PAs. Unless 

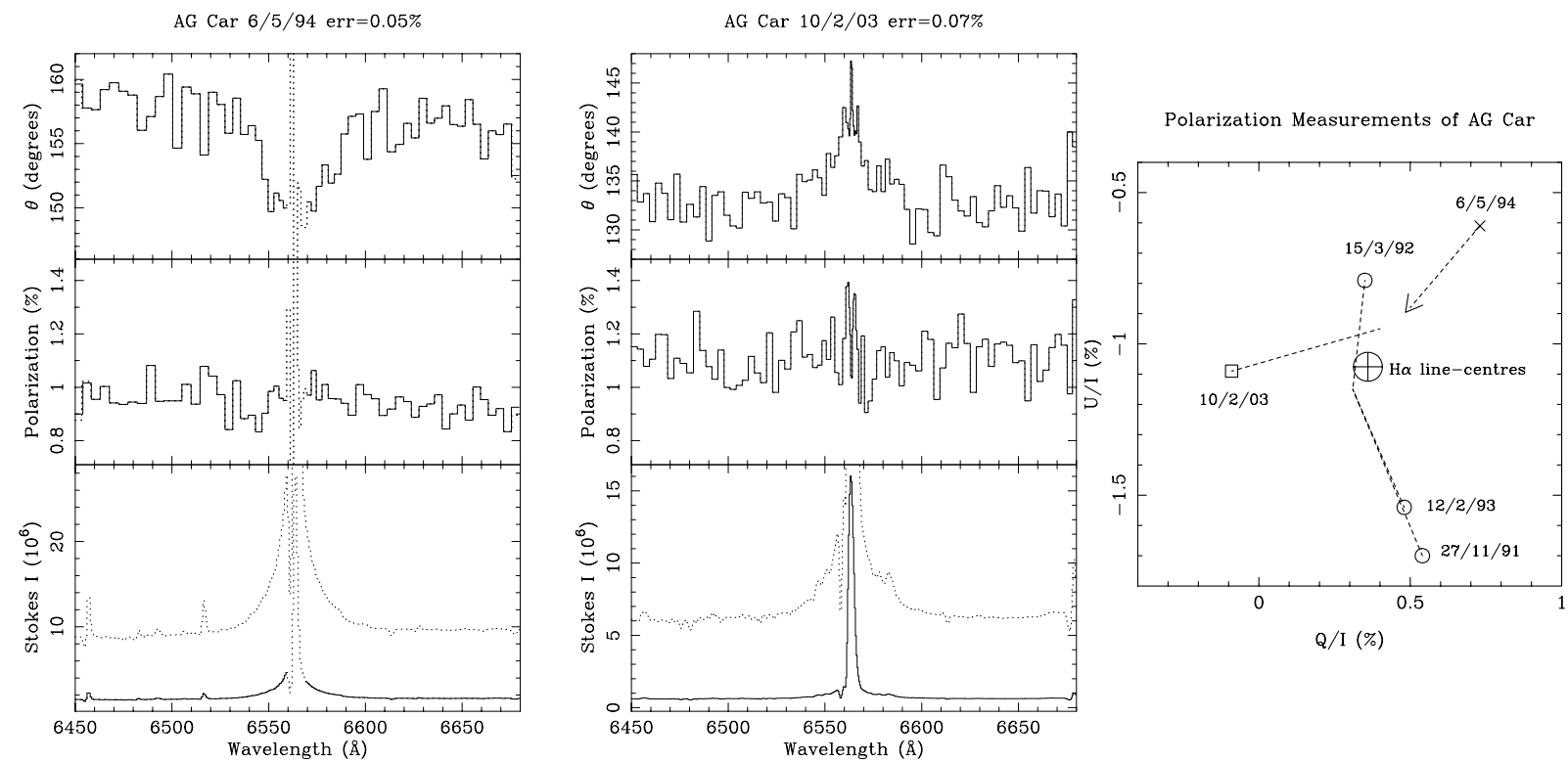

Fig. 3. Left, centre: polarization spectra of AG Car from the two dates observed. The bottom panel of each triplot shows the Stokes $I$, or intensity spectrum; and the middle and top panels show the degree and PA of the polarization respectively as a function of wavelength. The data has been rebinned to the $1 \sigma$ error indicated above each plot. The 6/5/94 data displayed erratic behaviour around the absorption component and line-centre due to reasons explained in Sect. 2, and was edited out of this plot for clarity. Right: multiple spectropolarimetric measurements of AG Car plotted in $Q-U$ space. Circles are continuum points from SL94; the cross is from archival data; and the square is our continuum data. Dotted lines show the excursions to the $\mathrm{H} \alpha$ line-centre polarization. Note that the 1994 excursion was attenuated due to fluctuating data (see section on AG Car), and that SL94 averaged their three line-centre polarizations together. The mean of the line-centre polarizations is marked on the plot, with a $1 \sigma \mathrm{rms}$ error of the size of the symbol. Uncertainties in the continuum are limited by the external errors, of order $0.1 \%$.

the assumption used to estimate the ISP are invalid, the evidence points away from the flip-flopping axi-symmetric wind scenario. Instead, it may point towards a "clumpy" wind model, where the PA of the intrinsic polarization at any time is related to the projected angle between the star and the dominant clump(s). This is the same scenario to that proposed for P Cyg by Nordsieck et al. (2001, see section on P Cyg).

HR Car Like AG Car, HR Car has been strongly variable for over 25 years ( $~ 21 \mathrm{mag}$ on timescales of $\sim 3$ years). It is surrounded by a filamentary bi-polar nebula $\left(\mathrm{PA} \sim 135^{\circ}\right)$ which has a kinematic age of $\sim 5000$ years (Nota et al. 1997). The star was noted by Serkowski et al. (1975) as polarimetrically variable, implying it has intrinsic polarization. This was confirmed by subsequent polarimetric monitoring by Parthasarathy et al. (2000). It was observed spectropolarimetrically by Clampin et al. (1995), who found evidence for intrinsic polarization at a PA of $30 \pm 1^{\circ}$, which is aligned with the "finger" of nebular emission $\left(\mathrm{PA} \sim 35^{\circ}\right)$ within $\sim 3^{\prime \prime}$ of the central star noted in their coronagraphic image. They also noted the presence of a number of clumps of emission close to the star, which did not correspond to the filamentary structures of the nebula and appeared to be roughly spherically distributed around the star.

In our spectropolarimetry, both epochs show line effects (see Fig. 4). The 1992 data shows a depolarization accompanied by a PA rotation. The 1992 intrinsic polarization is $P=0.67 \pm 0.03 \%, \theta=138 \pm 2^{\circ}$, which is aligned parallel to the major axis of the bi-polar nebula. The 2003 data shows a lower continuum polarization, with an increase in polarization across the emission line. The 2003 intrinsic polarization is $P=0.56 \pm 0.03 \%, \theta=13 \pm 2^{\circ}$. This PA bears no obvious relation to the PA measured at the previous epoch, nor to any large-scale nebular features.

The line-centre polarization from our observation, the archival data and the data in Clampin et al. (1995) agree well with each other, indicating that this is a good estimate of the ISP towards HR Car at $6563 \AA$ (as with AG Car). The mean of these measurements is $Q=-0.80 \pm 0.05 \%, U=-3.56 \pm$ $0.03 \%$; implying $P=3.66 \pm 0.05 \%, \theta=128.7 \pm 0.4^{\circ}$ (see Fig. 4, right).

Line-effects are also seen across Fe II lines at both epochs. In the 1992 data, there is a borderline detection across the $\lambda 6456$ line, with a line-to-continuum PA of $\sim 140^{\circ}$, i.e. parallel to the $\mathrm{H} \alpha$ depolarization. In the 2003 data, line-effects are detected across the Fe II $(\lambda \lambda 6417,6433,6456,6516)$ lines. The depolarizations all have PAs between $35-60^{\circ}$, but these are borderline detections.

Taking the ISP to be that calculated above we find that the Clampin data and archival data, taken approximately 1 year apart, are perpendicular to each other with PAs of $\sim 45^{\circ}$ and $\sim 135^{\circ}$ respectively. These align with the major and minor axes of the bipolar nebula. Our data, taken 10 years later, has a completely different PA of $\sim 15^{\circ}$. Taking the spectropolarimetry of Clampin et al. (1995) and multiple $R$-band polarimetric measurements of Parthasarathy et al. (2000) into account, the intrinsic polarization of HR Car seems to have no preferred axis with time. In a clumpy wind we may expect this kind of temporal variability, where the polarization at each epoch is related 

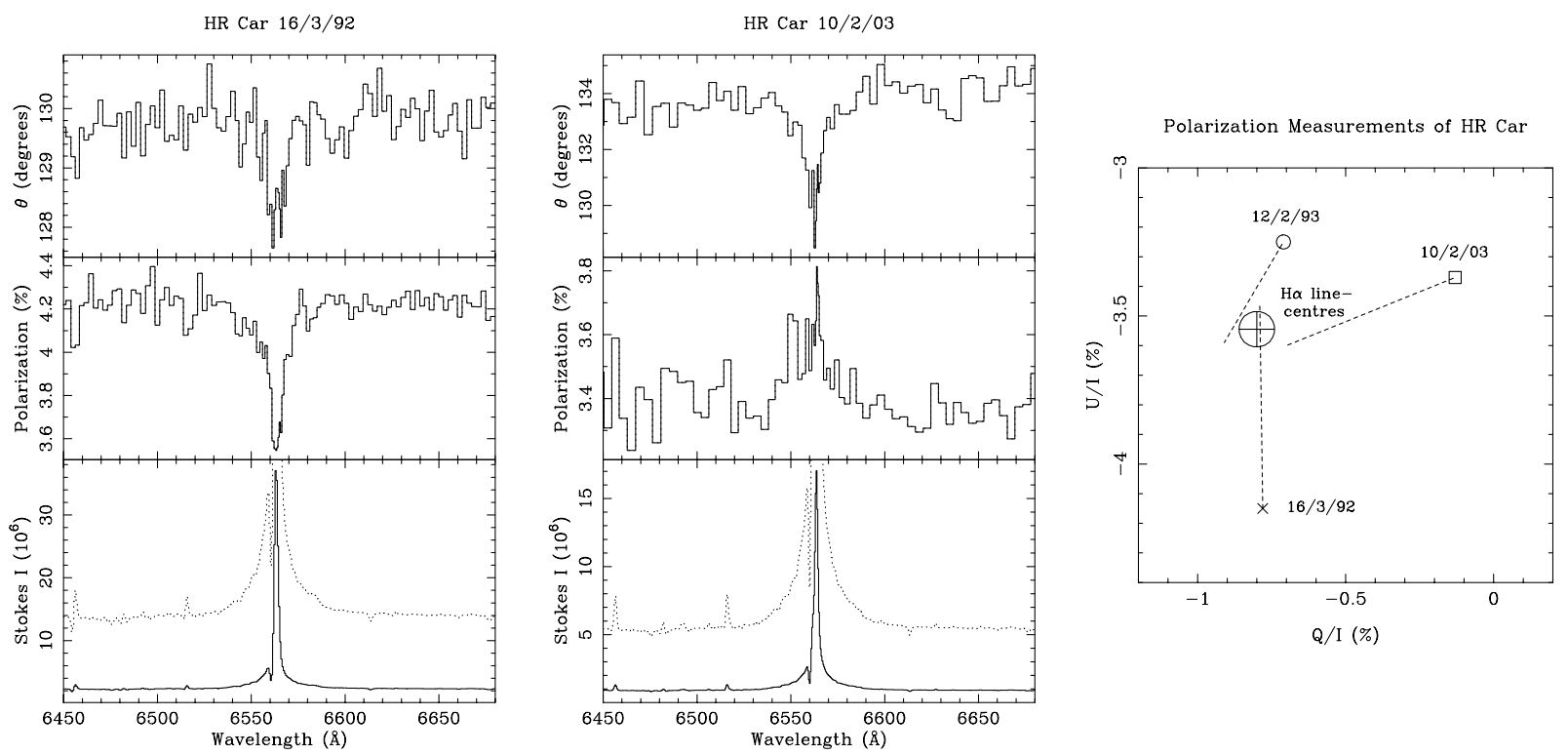

Fig. 4. Left, centre: polarization spectra of HR Car from the two dates observed. The panels show the same as Fig. 3, and the data has been rebinned to a $1 \sigma$ error of $0.05 \%$. Right: multiple spectropolarimetric measurements of HR Car, plotted in $Q-U$ space. The cross marks the continuum polarization at $\mathrm{H} \alpha$ for the 1992 archival data; the circle for the data taken by Clampin et al. (1995); and the square for our data. The dotted lines show the excursions to the line-centre polarization. The mean of the line-centre polarizations is marked, with a $1 \sigma$ rms error of the size of the symbol. Uncertainties in the continuum are limited by the external errors, of order $0.1-0.2 \%$.

to the projected PA between the star and the closest, densest clumps.

$\eta$ Car The most famous of the LBVs underwent a huge eruption in the mid-19th century when it increased in brightness by over 2 mags and ejected $\sim 10 M_{\odot}$ (Morris et al. 1999; Smith et al. 2003b), the product of which is the "homunculus" nebula we see today (for an extensive review of this object see Davidson \& Humphreys 1997). Aside from a smaller eruption in the late 19th century, which is possibly responsible for the mini-homunculus within the main nebula detected by Ishibashi et al. (2003), the star has been more or less stable. The periodic ( 2000 days) spectroscopic events are attributed to the star being in a highly eccentric binary system (Damineli et al. 2000); and the gradual brightening in the optical and NIR is attributed to the expansion of the nebula (Davidson \& Humphreys 1997). $\eta$ Car's wind as a function of latitude was studied by Smith et al. (2003a) by looking at starlight reflected of the surrounding nebula. They determined that the wind has higher terminal velocities and higher mass-fluxes towards the poles, assuming the star's axis is aligned with the bipolar nebula. Inteferometric observations by van Boekel et al. (2003) revealed that the star's NIR emission is prolate, with a major axis of PA $134 \pm 7^{\circ}$. This is aligned with the PA of the humunculous nebula (PA $132^{\circ}$, Morse et al. 1998).

Our data shows a very complex polarization profile, corresponding to an emission line which is made up of three components - the broad wings, the narrower component containing the bulk of the flux, and the narrow peak (see Fig. 5). The broad wings correspond to the $Q-U$ excursion labelled 1 with a PA of $91^{\circ}$; the flux-bulk to the excursion labelled 2 with a PA of $52^{\circ}$; and the narrow peak to the $Q-U$ loop marked 3, with a PA of $95^{\circ}$. There is also a loop as the vectors return from the highflux region to the continuum region. This does not correspond to any detectable spectral features.

Comparison of our spectrum with archive data taken by the Hubble Space Telescope (HST) one day previously reveals that the narrow emission peak (\#3) does not originate from the star, but from a nebulous region approximately 1" away. Our data does not spatially resolve the star and the nebula, and so this nebulous emission will not have been removed at the sky-subtraction stage of data reduction (see Sect. 2). The [N II] emission line redward of the $\mathrm{H} \alpha$ line, which displays a depolarization, also originates from the nebula. As the nebula is located well outside the polarizing region close to the star, any polarization it may have will be unrelated to that of the continuum. Closer inspection of the HST spectrum reveals that the nebula also has continuum emission, probably from reflected starlight.

Given that the light we see in our spectrum is made up of a direct starlight component and a reflected nebula component, these may be responsible for the distinct $Q-U$ excursions. The emission component containing the bulk of the flux ("2" in Fig. 5, which probably corresponds to a line-forming region close to the star) with a PA of $52 \pm 3^{\circ}$ is perpendicular to the major-axis of the resolved prolate structure detected by van Boekel et al. (2003). This is consistent with polarization due to scattering off a bipolar wind, as per the scenario suggested by Smith et al. (2003a). The level of depolarization, at $\sim 1 \%$, is also consistent with such a scenario (Cassinelli et al. 1987). The other $Q-U$ excursions, which seem to have PAs of $\sim 95^{\circ}$ and a much stronger depolarization of $\gtrsim 2 \%$, may be due to scattered light off the unresolved nebulous component. This hypothesis is supported by other sharp features of the spectrum 

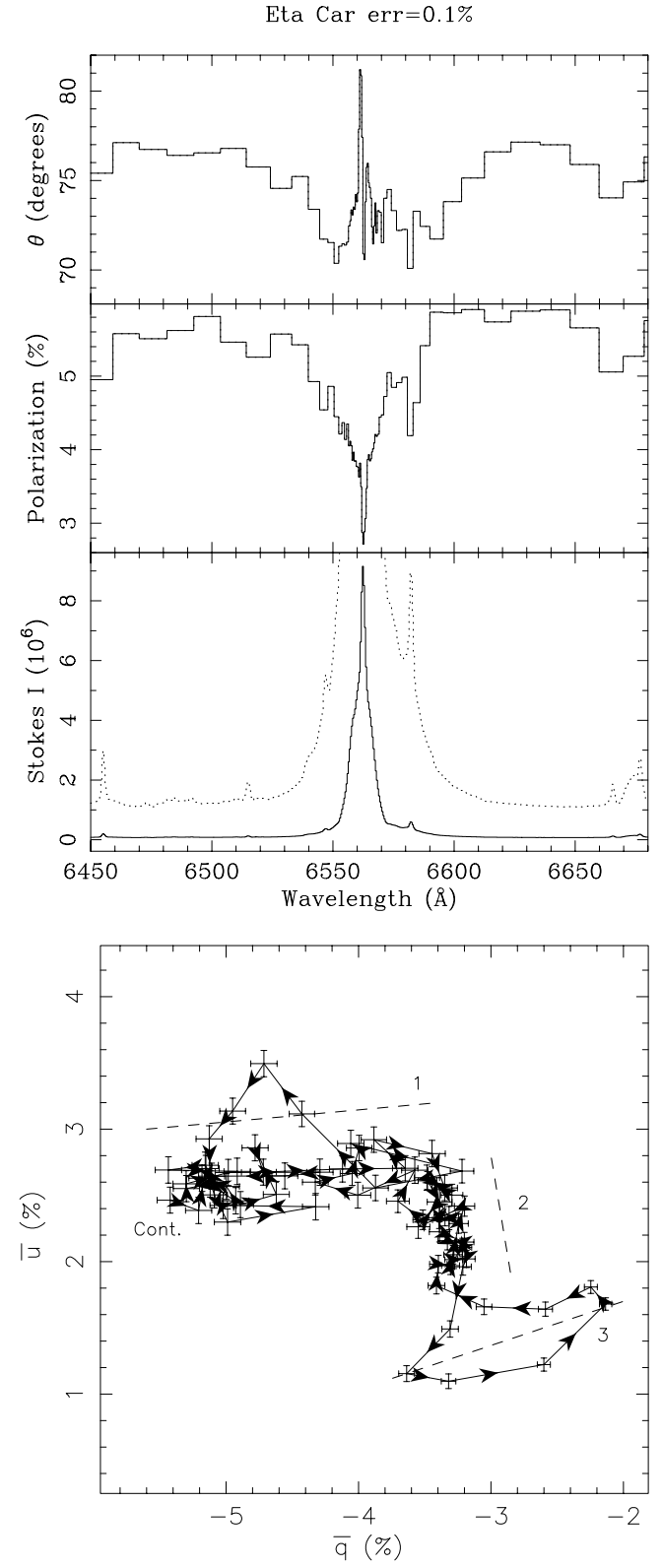

Fig. 5. Top: the polarization spectrum of $\eta$ Car. The panels show the same as Fig. 3. The data has been rebinned to a $1 \sigma$ error of $0.1 \%$. The dotted line shows a magnification of the Stokes $I$ spectrum to highlight the broad wings and weaker spectral lines. Bottom: the spectrum plotted in $Q-U$ space. The arrows join points of increasing wavelength. The location of the continuum is marked "cont.". The distinct excursions mentioned in the text are indicated with dotted lines. The excursion associated with the wings is marked "1"; the excursion associated with the narrower region containing the bulk of the line-flux is marked "2"; and the loop associated with the narrow, unresolved nebular emission is marked " 3 ".

(not shown here), which also show depolarizations with similar PAs of $\sim 90^{\circ}$. These PAs do not relate to any nebular features nor to the PA of the slit, but as shown in Schulte-Ladbeck et al. (1999) the polarization of $\eta$ Car varies greatly within $2^{\prime \prime}$ of the central source.

In summary, the complex polarization profile may be a combination of spatially unresolved emission from the nearby reflection nebula and emission from the stellar wind. The polarization of the light from the stellar wind is perpendicular to the bipolar axis of the homunculus nebula. This is consistent with single scattering off an optically thin bipolar wind, or multiple scattering off an optically thin equatorially-enhanced wind (Angel 1969; Wood et al. 1996).

Hen 3-519: this object is classified as a post-LBV/pre-WR star by (Davidson et al. 1993). It is not observed to be variable and has broad emission-lines in its spectrum. For these reasons it is not possible to determine the star's temperature at observation using the two methods outlined in Sect. 3.1. The star is surrounded by a roughly circular nebula of diameter $\sim 55^{\prime \prime}$. The nebula appears to be a clumpy bubble, rather than a detached ring as seen around AG Car (Stahl 1987; Duncan \& White 2002). The spectra from the two different epochs separated by $\sim 9$ years look very similar, with the exception of a P Cygni profile present in the $\mathrm{H} \alpha$ line of the Feb. 2002 data not present in the 1994 data.

The archive data from 6/5/94 (Fig. 6, left) does not show an obvious line-effect. The data from 10/2/03 shows different polarization behaviour across the features of the line (see Fig. 6 , centre). The line-centre polarization is consistent with that of the archival data, showing up in $Q-U$ space as a dark cluster of points above the continuum region. This behaviour is also seen in the HeI $\lambda \lambda$ 5876, 6678 lines. The mean line-to-continuum vector, and hence intrinsic polarization, is $Q=-0.10 \pm 0.03 \%$, $U=-0.14 \pm 0.03 \% ; P=0.17 \pm 0.04 \%, \theta=117 \pm 12^{\circ}$. The mean of the line-centre polarizations is $P=2.89 \pm 0.02 \%$, $\theta=97.9 \pm 0.3^{\circ}$, which agrees with the data of the previous epoch.

As well as the $\mathrm{H} \alpha$ line having a slightly different polarization to the continuum, there is a large change in polarization in the blue wing of the 2003 data, showing up as a looped excursion in $Q-U$ space (see Fig. 6, right). This behaviour in the blue-wing is also seen in the He I $\lambda \lambda$ 5876, 6678 lines. The mean change in polarization from the line-centre polarization of these loops is $P=0.87 \pm 0.08 \%, \theta=25 \pm 3^{\circ}$. At present, we have no explanation for these features, which cannot be due to the simple dilution of the polarized continuum photons. They are also unlikely to be related to the "McLean" effect (McLean 1979), where an increase of polarization due to scattered light is seen across blueshifted P Cygni absorption (see Vink et al. 2002), as here the features in Hen 3-519 extend beyond the blueshifted part of the P Cygni absorption.

WRA 751: this star was first considered to be an LBV by $\mathrm{Hu}$ et al. (1990), but is still referred to as an LBV candidate by many authors. It is classified as a strong-amplitude variable by VG01, but the AAVSO light-curve shows that it has not varied by more than $0.5 \mathrm{mag}$ over the last $\sim 10$ years. Radio images by Duncan $\&$ White (2002) show a roughly circular, clumpy nebula of diameter $\sim 20^{\prime \prime}$ with evidence for a central, edge-on nebular ring of diameter $\sim 5^{\prime \prime}$. The object's polarization spectrum shows no line-effect (see Fig. 7).

If the light from the star is intrinsically polarized, the upper limit we can put on this is rather high due to the low $S / N$ 

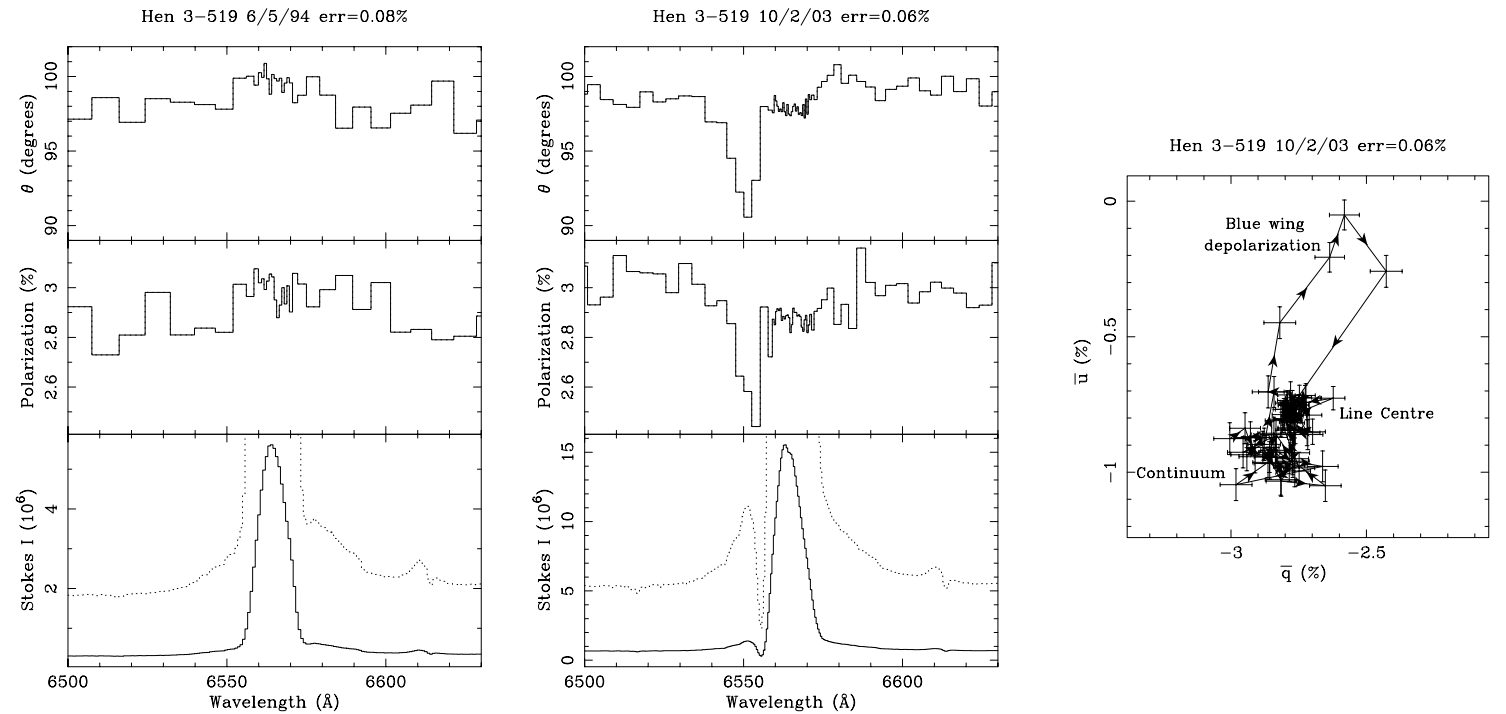

Fig. 6. Left, centre: the polarization spectra of Hen 3-519 from 6/5/94 and 10/2/03 repectively. The panels show the same as Fig. 3 and the data has been rebinned to the $1 \sigma$ error indicated above each plot. Right: the polarization spectrum of the 10/2/03 data plotted in $Q-U$ space. Arrows join points of increasing wavelength. The distinctly separate continuum and line-centre regions have been marked, as well as the loop corresponding to the depolarization seen in the blue wing.

of the spectrum and the relative weakness of the $\mathrm{H} \alpha$ line (see Sect. 3.2, Eq. (4)). The sensitivity limit for detection of dilution of the continuum by the emission line is $0.44 \%$. As we have positive detections in this study of less than $0.3 \%$, we cannot rule out a similar level of asphericity in WRA 751's wind.

P Cyg: since undergoing two huge eruptions in the 17th century, there is no evidence this star has undergone any shorttimescale variability, although de Groot \& Lamers (1992) argue the star has gradually brightened by about 0.5 mag since then as it evolves redward. The star's nebula has been the subject of much investigation over the last $\sim 10$ years. It consists of an inner- and outer-shell, of diameters $22^{\prime \prime}$ and $1.6^{\prime}$ respectively, both with roughly spherical morphologies (Barlow et al. 1994; Meaburn et al. 1996). Optical images of the circumstellar material reveal a complex clumpy morphology down to the inner 0.2" (Meaburn et al. 2000; Chesneau et al. 2000). Multiple interferometric radio observations have shown that the clumpiness of the inner $0.4^{\prime \prime}$ of the nebula is highly variable on timescales of days (Exter et al. 2002).

Both the observations of this object, taken 3 days apart, show changes in polarization just blueward of the centre of the emission line, with varying behaviour from set to set. They line up not with the P Cygni absorption component but with the steep rise in flux from the absorption component to the centre of the emission line, which may be at the limit of the instrument's spectral resolution. As polarization in this part of the spectrum varies wildly from set to set, whilst the continuum level remains constant, we therefore explain these features as artifacts introduced by the slightly different spectral resolutions of the $O$ and $E$ beams emerging from the calcite. In terms of difference in polarization between the continuum and the line-centre, there is no change within the uncertainties (see Fig. 7). The detection limit is $0.15 \%$ and $0.18 \%$ for the $10 / 12 / 03$ and $13 / 12 / 03$ observations respectively. The $\mathrm{H} \alpha$ line-centre polarization we measured agrees well with the line-centre polarization measured by Taylor et al. (1991) and Nordsieck et al. (2001), supporting the hypothesis that the $\mathrm{H} \alpha$ line is intrinsically unpolarized. We find no change in continuum polarization for our two datasets taken 3 days apart.

Multiple spectropolarimetric measurements of P Cyg are described by both Taylor et al. (20 measurements) and Nordsieck et al. (15 measurements). Both found that while the line-centre polarization remained constant the continuum polarization was variable on timescales of days. Both interpreted this as variable intrinsic polarization of the object coupled with the ISP, with an intrinsically unpolarized $\mathrm{H} \alpha$ line as described above for AG Car and HR Car. Taylor et al. found that the intrinsic polarization varied from $0.04 \%$ to $0.48 \%$ at random PAs. Such behaviour was ascribed by Nordsieck et al. to electron scattering by a clumpy wind, with a spherical distribution of clumps and a clump-ambient material density contrast of $\gtrsim 20$. It may be the case that at the epoch of our observations any intrinsic polarization due to wind clumps was too small to be detected.

HD 160529: despite being established as spectroscopically and photometrically variable for over 30 years (Wolf et al. 1974), Serkowski et al. (1975) noted no polarimetric variability, and it is listed by Hsu \& Breger (1982) as a "polarized standard". This star represents the lower-luminosity end of the LBV instability strip and is not strongly variable, with photometric variations of only a few tenths of a magnitude over the last 20 years (Sterken et al. 1991; Stahl et al. 2003). The star has no associated nebulosity, no nebulous emission in its spectrum and no evidence of circumstellar dust (Hutsemekers 1997; Nota \& Clampin 1997). We note no [N II] emission in our spectra. 

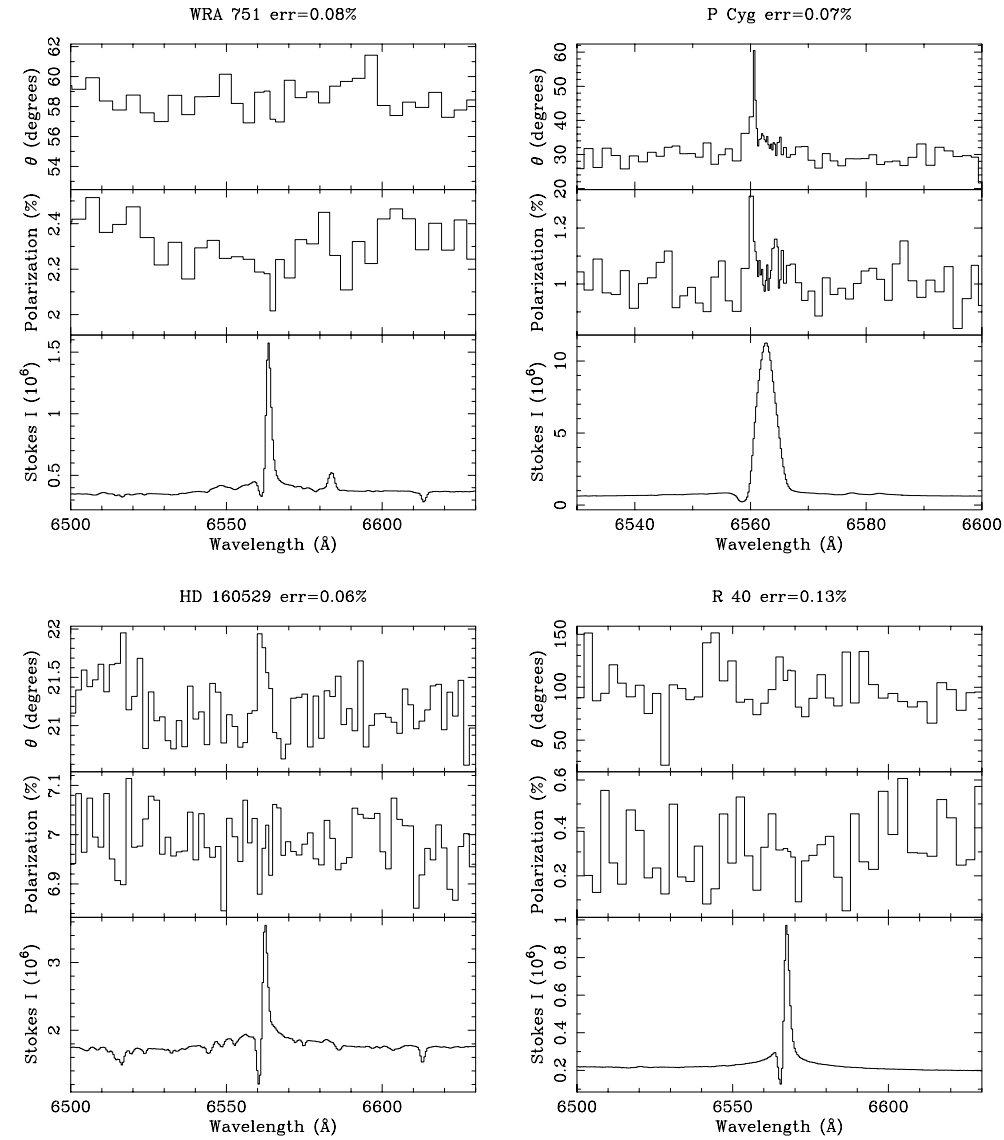

Fig. 7. Polarization spectra of the Galactic LBVs WRA 751, P Cyg and HD 160529; and the SMC LBV R 40. The panels show the same as Fig. 5, and the data has been rebinned to the $1 \sigma$ errors stated above each plot. Note the different wavelength range of $\mathrm{P}$ Cyg. The jump in polarization seen in the $\mathrm{H} \alpha$ absorption component of $\mathrm{P} \mathrm{Cyg}$ is attributed to the steep increase in Stokes $I$ being unresolved, as explained in Sect. 2. HD 160529 did not display a line-effect at either epoch it was observed, and only the Feb. 2003 data is shown here.

The relative weakness of the $\mathrm{H} \alpha$ emission makes the detection of a line effect difficult for this object (Fig. 7). The detection limit for a line-effect for this observation is $0.4 \%$.

R 40: this star is the only known LBV in the SMC. Sterken et al. (1998) show that R 40 increased steadily in brightness by 0.7 mag in the years 1985-1995. AAVSO records show this object has been constant in luminosity since $\sim 1995$, although there is a gap in their records of $\sim 3$ years (Waagen 2005). There is presently no evidence for associated nebular or dust emission, and we find no [N II] emission in its spectrum.

No line-effect was observed on either of the observing dates. As the object was observed on consecutive nights and the data for each night looked the same, the data were combined to improve the $S / N$, but still no line-effect was observed (Fig. 7). The detection limit is $0.37 \%$.

S Dor: despite being one of the "archetypal" LBVs for many years, the star has only recently been observed with the spectral appearance of an F-type supergiant - the common spectraltype of LBVs at visual maximum (Massey 2000). Also, it has no directly detectable LBV nebula. Weis (2003) found that the dynamical age of the large-scale elliptical nebula surrounding $\mathrm{S}$ Dor meant that it was too old to have been formed in the LBV phase; and despite broadened [N II] emission in the star's spectrum (also noted in our spectrum), any circumstellar nebula from which it originates would have to be less than the detection limit of $\sim 0.25 \mathrm{pc}$ in diameter. The star's spectrum indicates a $T_{\text {eff }}$ at observation of $\sim 9000 \mathrm{~K}$, putting the star close to visual maximum. The bolometric correction (BC) method of determining the star's temperature is unreliable, due to disagreement over the star's temperature and luminosity at visual minimum, and also that the star may vary in bolometric luminosity during outbursts (see VG01). The AAVSO light-curve of S Dor shows it to have been exhibiting strong-amplitude variability over the last $10-15$ years.

The polarization spectrum (Fig. 8) has a suggestion of a broad PA rotation across the line from $\sim 60^{\circ}$ to $\sim 40^{\circ}$. This corresponds to a $2 \sigma$ detection and is therefore a borderline case. The single pixel dip in PA at the centre of the line is not trusted as "real" behaviour, as sharp spectral features can produce erratic data for the reasons described in Sect. 2. The detection limit in this data is $0.2 \%$.

R 71: the star is noted by Weis (2003) as having no visually detectable nebula and no nebular emission in its spectrum, although Voors et al. (1999) did find evidence for a warm dust component. Also, our spectrum does show very weak [N II] $\lambda 6584$ emission which suggests a nebular component. VG01 states that this star has never been observed as cool as the classical LBV minimum temperature of $\sim 8500 \mathrm{~K}$. AAVSO records show this star has been constant in brightness to within $\sim 0.2$ mag for the last $\sim 15$ years, although the star did increase in brightness by 0.5 mag for about a year in 2003 (Waagen 2005). 

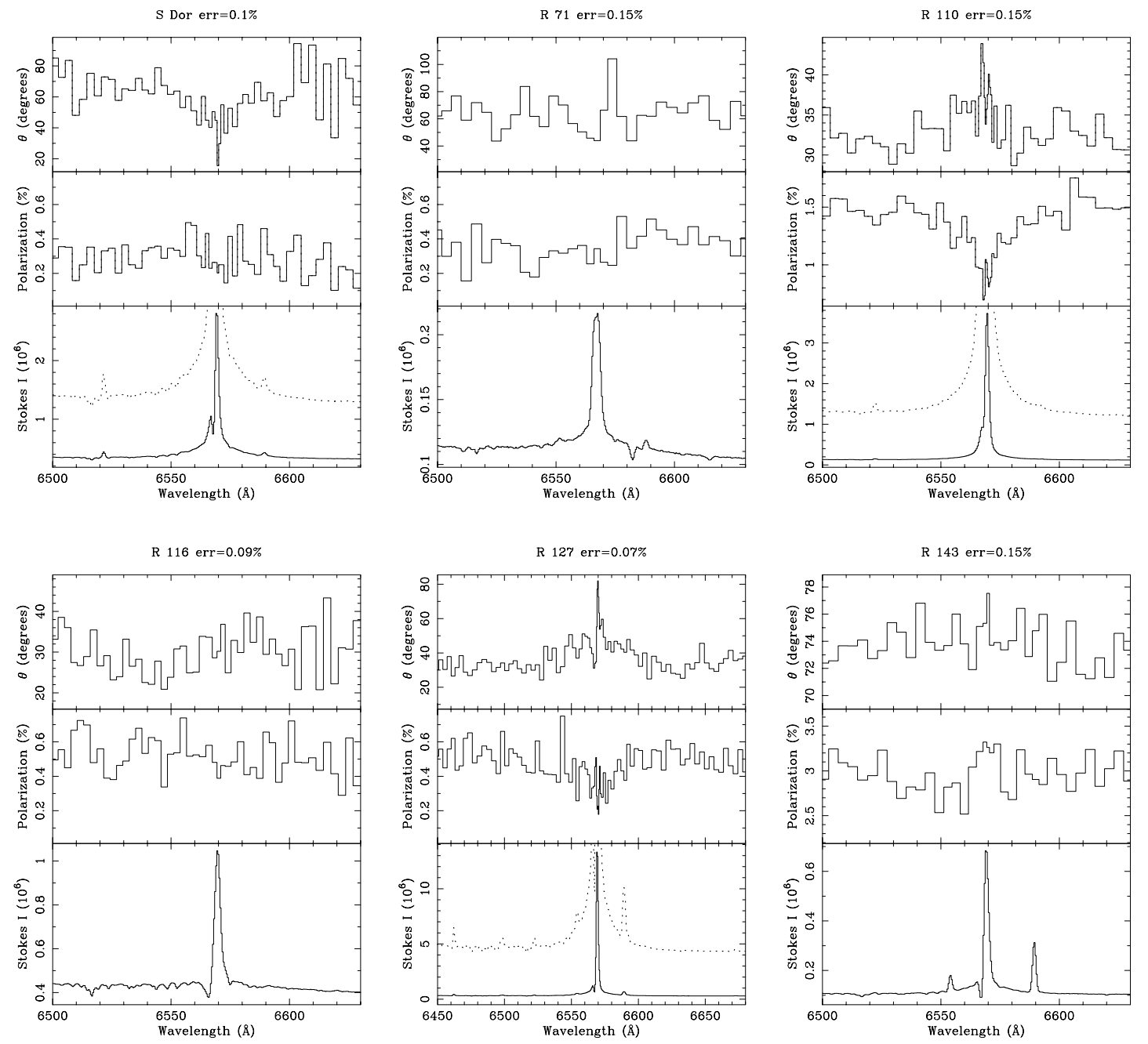

Fig. 8. Polarization spectra of the LMC LBVs S Dor, R 71, R 110, R 116, R 127 and R 143. The panels show the same as Fig. 7. Note the different wavelength scale for R 127 . Where line-effects are observed, the Stokes $I$ spectrum is magnified to show the broad change in polarization associated with the wings of the emission line. A narrow P Cygni absorption component in the $\mathrm{H} \alpha$ line of $\mathrm{R} 127$ was found to introduce artifacts in the polarization spectrum (see Sect. 2), and has been edited out.

This object shows no line-effect (Fig. 8). The comparitive weakness of the $\mathrm{H} \alpha$ line in this data means that the detection limit is $0.4 \%$.

R 110: it is not known whether this star has associated circumstellar dust or gas as it is poorly-studied. We do find, however, weak [N II] $\lambda 6584$ emission in our spectrum, which suggests that the star may have some form of nebula. The star was observed to increase in brightness by 0.9 mags to $m_{\mathrm{V}}=9.7$ between 1960 and 1993 by van Genderen et al. (1997). AAVSO photometric data on this object is limited, but it has been seen to decrease in brightness to $m_{\mathrm{V}} \sim 11$ in the last $\sim 10$ years (Waagen 2005).

The data exhibits a broad depolarization of $\gtrsim 0.5 \%$ accompanied by a broad PA rotation (Fig. 8). The width of this feature corresponds to the width of the broad wings of the emission line. The sharp features observed in the polarization spectrum at the line centre are spread over $\sim 10$ pixels, which imply that they are resolved and are judged to be real.
The line-to-continuum vector in $Q-U$ space yields an intrinsic polarization of $(0.55 \pm 0.09 \%)$ at a PA of $(26 \pm 5)^{\circ}$.

R 116: the similarities between this star and $\zeta_{1}$ Sco noted by van Genderen \& Sterken (1999) led them to suggest that R 116 is an ex/dormant-LBV. AAVSO records show that the star has been constant in brightness over the last 15 years to within 0.2 mag (Waagen 2005), and photometry collated from various sources by van Genderen \& Sterken suggested the star has been constant for over 40 years. There is presently no evidence for associated nebular or dust emission.

This object shows no line-effect (Fig. 8). The detection limit is $0.28 \%$.

R 127: the LBV nature of this star was discovered by Stahl et al. (1983). Since then it has been observed to cool to the classical LBV minimum temperature of $\sim 8000 \mathrm{~K}$ around 1993 , and is now apparently on its way back to visual minimum (VG01 and references therein; Waagen 2005). Coronagraphic imaging by Clampin et al. (1993) revealed an axisymmetric 
circumstellar nebula, with density enhancements either side of the star at a PA of $\sim 100^{\circ}$ and an opening angle of $90^{\circ}$. HST observations by Weis (2003) reveal evidence for asymmetric expansion within the inner nebula.

Spectropolarimetry by Schulte-Ladbeck et al. (1993) revealed a high degree $(\sim 1 \%)$ of intrinsic, variable polarization; the PA of which remained roughly perpendicular to the density enhancements. They explained the roughly constant intrinsic PA and observed P Cygni profiles as electron-scattering off an expanding equatorially-enhanced wind aligned with the outer nebula. The variable degree of polarization and slightly varying PA were explained by a clumpiness of the wind.

Our data shows a broad rotation in PA which corresponds to the broad wings of the emission line (Fig. 8). The emission line has a narrow P Cygni absorption component which was found to introduce erratic behaviour in the polarization spectrum. For the sake of clarity this feature was edited out. The sharp increase in PA at the line-centre is spread over $\sim 5$ pixels and so are judged to be real. The line-centre polarization has been attenuated slightly by editing out the absorption component, but the $Q-U$ excursion approaches the $\mathrm{H} \alpha$ line-centre polarization measured by Schulte-Ladbeck et al. (1993).

Taking this to be the ISP towards R 127, the intrinsic polarization is $0.50 \pm 0.04 \%$ at a PA of $20 \pm 2^{\circ}$. This is in good agreement with Schulte-Ladbeck et al. who found a PA of $24.4^{\circ}$ in the $V$ band, although at a higher degree of polarization of $1.37 \%$. These results, along with other polarization measurements collated by Schulte-Ladbeck et al., show that the intrinsic polarization of $\mathrm{R} 127$ appears to be at a roughly consistent PA of $25 \pm 10^{\circ}$. This endorses their proposed model of an equatorially-enhanced, clumpy wind viewed at a high inclination angle.

$R$ 143: Weis (2003) reported that the star is located in a small, triangular-shaped nebula of linear size $\sim 1.2 \mathrm{pc}$ within the 30 Dor region. The disrupted shape of the nebula was speculated by Weis to be due to strong stellar winds, high density and turbulent motions present in 30 Dor. When observed by Nota et al. (1995), the nebula was found to have filamentary structures eminating from the central source, reminiscent of HR Car. However, Smith et al. (1998) found that the dynamics of these structures suggested they were not associated with the star but of the background 30 Dor H II region. They did find evidence for a small clump of ejected material within $2^{\prime \prime}$ of the star but could not draw any conclusions as to its morphology.

This object shows no line-effect (Fig. 8). The detection limit is $0.34 \%$ due to poor $S / N$.

\section{Discussion}

Of the 11 confirmed LBVs, we have 5 positive detections of line-effects plus the borderline case of S Dor. Of the three LBV candidates, only Hen 3-519 shows a change in polarization across its emission lines, and the behaviour of this star's polarization spectrum cannot simply be explained by the dilution of the polarized flux by the emission line. Of the non-detection objects, in some cases the detection limits exceed the levels of depolarization observed in objects with line-effects. There may therefore be similar levels of depolarization in some of these objects that have gone undetected. Even if all LBVs had aspherical winds, we would expect some non-detections due to inclination and cancellation effects e.g. face-on disk, or two clumps at $90^{\circ}$ to each other. We therefore put the rate of windasphericity in LBVs at $\gtrsim 50 \%$, higher than that of the other classes of evolved massive star that have been studied comprehensively, $\mathrm{O}$ supergiants and Wolf-Rayet stars (25\% and 20\% of sample sizes 20 and 16 respectively, Harries et al. 2002, 1998).

Rotation and mass loss play intricate roles in the evolution of massive stars (Langer 1997; Maeder \& Meynet 2000). Given the transitional role of LBVs within the evolutionary paths of massive stars, the high incidence of intrinsic polarizations that we find in comparison to those of $\mathrm{O} / \mathrm{WR}$ stars is expected to reveal telling information about the onset of wind asymmetries. To probe the origin of the wind asymmetries, we correlate our detections with a range of LBV parameters (Sect. 4.1), before we discuss possible scenarios in Sect. 4.2.

\subsection{Correlations}

In terms of temperature and phase, no correlation is observed between detections and non-detections - line-effects are observed at visual minimum (HR Car), maximum (R 110), and intermediate (e.g. R 127) phases. No correlation is observed with luminosity, although we point out that the luminosities of these objects (even those in the Clouds) are uncertain, with different authors stating different values for $L / L_{\odot}$. VG01 states that luminosities of Galactic objects are uncertain to $0.2-0.3$ dex, while MC objects are uncertain to $\sim 0.1$ dex.

\subsubsection{Line-strength - wind asphericity relation?}

Inspection of Table 4 and Fig. 9 shows that the objects showing line-effects all have strong $\mathrm{H} \alpha$ lines. Indeed, the strongest $\mathrm{H} \alpha$ line definitely showing no line-effect is $\mathrm{R} 143$, with a lineto-continuum contrast of 6.4. S Dor has a line contrast of 8.3, and has a borderline case for a line-effect. Only P Cyg of the strong-emission objects has no clear-cut line-effect. From this we could draw one of two conclusions: either the method is not sensitive enough to detect line-effects in weak emission lines; or objects with strong $\mathrm{H} \alpha$ lines have strong asphericity in their winds. Line-effects (albeit weak) are seen across the He I 16678 line in the spectrum of AG Car (contrast $\sim 1.7$ ), the weaker lines in $\eta$ Car's spectrum $(\sim 1.7)$, and the weak Fe II lines in the spectrum of HR Car $(\sim 1.5)$. This therefore leads us to discount the first explanation and suggest that LBV winds producing stronger emission are more likely to have an aspherical geometry.

\subsubsection{Galactic vs. extra-Galactic LBVs}

Of the Galactic objects observed, 4 out of 7 show line-effects. Of the three non-detections, one (P Cyg) has been shown to exhibit a change in polarization across its emission lines at earlier epochs (Taylor et al. 1991; Nordsieck et al. 2001). 


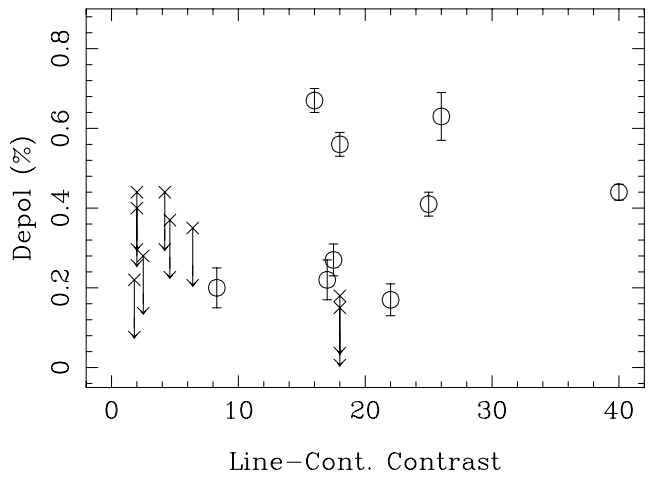

Fig. 9. Strength of depolarization against the line-to-continuum contrast of the $\mathrm{H} \alpha$ line. Circles show line-effects, crosses show nondetections. Where no depolarization was observed, the upper limit for an undetected line-effect is shown. For the sake of clarity, $\eta$ Car is not shown as its depolarization and line contrast are both much greater than any other object.

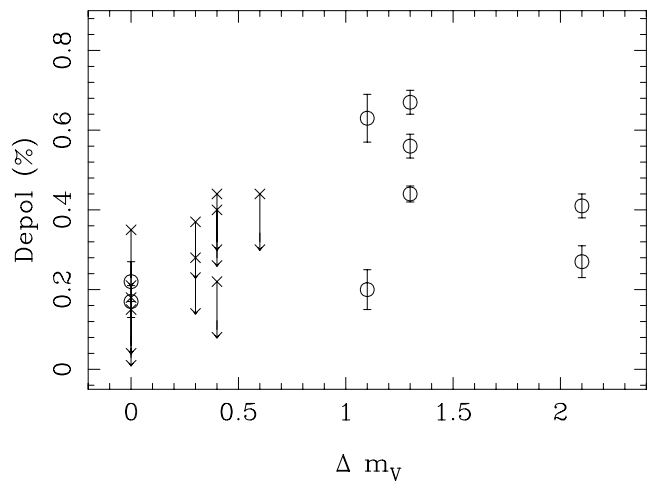

Fig. 10. Strength of depolarization against apparent variability over the last ten years. The symbols show the same as Fig. 9.

By comparison, only 2 out of 7 Magellanic Cloud objects show line-effects, with one borderline case (S Dor). One explanation for this difference may be that the selection of Galactic LBVs is not truly representative of the LBV phenomenon. Hen 3-519 is commonly thought-of as a post-LBV object, indeed its broad emission lines are reminiscent of a Wolf-Rayet star. WRA 751 is located in a distinctly separate location on the H-R diagram to the other LBVs (see Fig. 2) and has not been observed to show variability on the same scales as other LBVs such as AG Car or S Dor (van Genderen et al. 1992). $\eta$ Car and P Cyg are unusual objects even by LBV standards - whilst both had huge outbursts hundreds of years ago, P Cyg has been roughly constant in luminosity for many years, while $\eta$ Car's variability is often explained by the expanding nebula moving across our line of sight, coupled with the effects of being in a highly eccentric binary system (Israelian \& de Groot 1999; Davidson \& Humphreys 1997). This leaves AG Car, HR Car and HD 160529 as the only "normal" Galactic LBVs. We may expect the MC LBVs to be a more homogeneous group, but as the data quality for the MC LBVs is not as good as for the Galactic ones, and because of the low sample numbers involved, we draw no conclusions as to differences between these two populations at the current stage.

\subsubsection{Wind asphericity and strong-amplitude variability}

A correlation can be found by looking at recent light-curves of the objects. Observations from the last $\sim 15$ years obtained from the AAVSO and the Tycho mission show that, of the objects showing definite line-effects, all show variability of $\gtrsim 1 \mathrm{mag}$ (see Fig. 10). Of the others, only S Dor shows this kind of variability, and this object was a borderline detection. We therefore propose a link between wind asphericity and recent, strong variability. Figure 11 shows the light-curve of AG Car, going back 15 years. Marked on the plot are the H-D limit and the bistability jump (BSJ, see Sect. 1 and Lamers et al. 1995; Vink et al. 1999), under the assumption that the star varies at constant bolometric luminosity. It can be seen that AG Car has crossed its H-D limit and spent long periods very close to the BSJ during this time. The same is true for R 127 (Waagen 2005) and $\mathrm{S}$ Dor, although the assumption of constant bolometric luminosity may break down for S Dor (VG01). Whilst the luminosities of HR Car and R 110 mean they are below the H-D limit (leaving the uncertainties in their luminosities aside), both stars have shown recent strong variability (AAVSO, Waagen 2005), and are close to a second BSJ proposed by Lamers et al. (1995) at $\sim 10000 \mathrm{~K}$, as Fe III recombines to Fe II. Proximity to the BSJs coupled with a variable effective temperature may play a part in producing wind asphericity.

\subsection{Interpretation of polarimetric variability}

We have shown that at least half of the LBVs observed display evidence for intrinsic polarization. Of the 4 objects for which multi-epoch observations exist, all are polarimetrically variable, with both the degree of polarization and PA changing on timescales of $\sim$ a week to months. As the bulk of the polarization occurs within a couple of stellar radii, variable wind properties of an outflow moving at a few hundred $\mathrm{km} \mathrm{s}^{-1}$ could produce variability on these timescales. Here we will discuss four different possible scenarios for producing such behaviour.

\subsubsection{Flip-flopping wind}

In this scenario, the wind flips from an equatorially-enhanced flow to a bi-polar flow or vice versa. Such a scenario could occur if the star was close to a BSJ and was rotating close to break-up, producing a temperature differential between the equator and the poles. As the star's global $T_{\text {eff }}$ changes, one of these regions may reach the BSJ temperature before the other, changing the geometry of the wind. As the plane of the equator and poles are perpendicular to each other, at this point we may observe a $90^{\circ}$ flip in intrinsic polarization. At intermediate stages we would expect the polarization of the two components to cancel each other out, and so we would observe lower levels of polarization but at PAs more or less aligned with one of the two axes.

The results of SL94 showed that, for three separate observations of AG Car over 2 years, the PA of the intrinsic polarization flipped roughly by $90^{\circ}$ from $55^{\circ}$ to $133^{\circ}$ and back again. From Fig. 11 we can see that the star brightened by $\sim 0.2$ mag 
AG Car Light-Curve

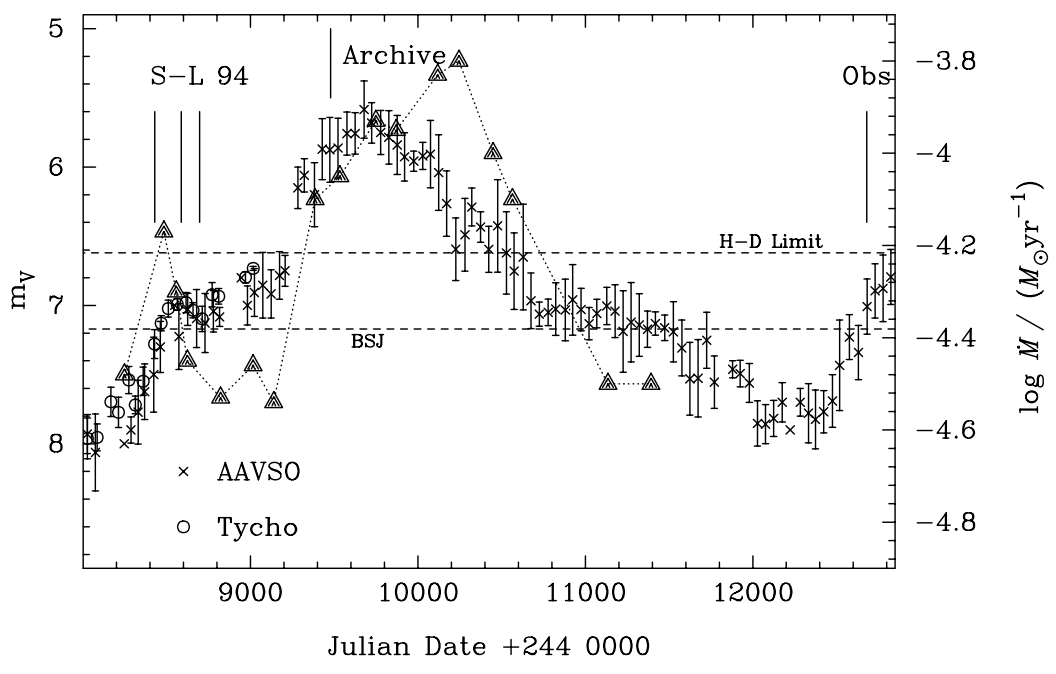

Fig. 11. The light curve of AG Car for 1989-2004. Circles show data from the Tycho mission, crosses show validated data from the AAVSO records. The data has been rebinned to 50 days, and the error bars show the $1 \sigma$ standard deviations. The dates of our observation, the archive observation, plus the observations of SL94 have been marked. Also marked are the brightnesses at which the star is crossing the empirical H-D limit, and the bi-stability jump (BSJ) at $\sim 21000 \mathrm{~K}$ (under the assumption that the star varies at constant bolometric luminosity). Also marked on the plot are the mass-loss rates derived by Stahl et al. (2001) (triangles).

between their first and second observation, then had dimmed by $\sim 0.1$ mag by the time of their third. We can also see that the star was close to its BSJ at this time, assuming the star varies at constant luminosity. This alone is consistent with a flip-flopping wind. However, when one looks at the PAs measured at 1994 $\left(26^{\circ}\right)$ and $2003\left(84^{\circ}\right)$, as well as those measured by Leitherer et al. (1994), these angles are completely unrelated; whilst the degree of polarization remains in the range $0.4-0.6 \%$. This suggests that the results of SL94 may have been a result of limited temporal sampling, and that the flip-flopping wind cannot be the sole explanation of the polarization behaviour. A similar situation is observed for HR Car and P Cyg, where the PA appears random with time and bears no relation to the level of polarization.

\subsubsection{Opacity changes within the wind}

As explained in Sect. 1, single scattering off a flattened wind would produce polarization perpendicular to the plane of scattering. However, if the opacity was to increase, multiple scattering effects would come into play. It has been shown by e.g. Angel (1969) and Wood et al. (1996) that multiple scattering in an optically thick, flattened wind can produce polarization parallel to the plane of scattering. If the density of the inner regions of the wind were to increase due to e.g. an increase in mass-loss rate, we may expect to see the PA of polarization change from perpendicular to parallel to the flattened wind's major axis. As with the flip-flopping wind, we would expect intermediate stages to have low degrees of polarization in one of the two planes due to cancellation effects.

In Fig. 11, the mass-loss rate for AG Car as determined by Stahl et al. (2001) are overplotted on the star's light curve. We can see that between the first two measurements by SL94, the mass-loss rate drops, coinciding with a $90^{\circ}$ flip in the star's intrinsic polarization. However, at the time of SL94's third observation, at which point the polarization had flipped back, the mass-loss rate had remained roughly constant. The seemingly unrelated PAs at similar levels of polarization of the later observations are further reason to disregard this scenario for AG Car. The random temporal behaviour of HR Car and P Cyg also cannot be explained this way.

\subsubsection{Clumpy wind}

Here, the wind is not a smooth homogeneous outflow but instead consists of localized density enhancements, or "clumps". The bulk of the scattering would be due to the closest, densest clumps. If the clumps were ejected in a spherical distribution, or in a flattened wind which was observed at a low inclination, the PA of the intrinsic polarization would be related to the projected angle between the star and the clump, and appear effectively random with time. Only in a flattened wind observed at a high inclination angle would the PA remain roughly constant. Multiple clumps within the wind could serve to increase the intrinsic polarization or cancel each other out, making the degree of polarization also seemingly random with time. Wind-clumping has previously been invoked to explain the random polarimetric variations observed in Wolf-Rayet (WR) stars (Robert et al. 1989).

AG Car, HR Car, and P Cyg are all described in this paper to have intrinsic polarization which appears to be essentially random with time, both in level and PA. This is consistent with the clumpy wind scenario, with the clumps ejected in a roughly spherical distribution on the plane of the sky. Only R 127 has polarization with PA which remains roughly constant at $25 \pm 10^{\circ}$. This is consistent with a clumpy wind ejected on a significantly flattened plane on the sky. The P Cygni profiles observed in the star's spectrum imply that material is being ejected towards us along the line-of-sight, therefore the favoured explanation of Schulte-Ladbeck et al. (1993) was that we are looking at an edge-on, equatorially-enhanced, clumpy wind. Our latest measurement supports this model.

The strength of the polarimetric variability of LBVs is greater than that observed in WRs (Robert et al. 1989). It is 
interesting to note that Robert et al. find that the amplitude of the variability in WRs increases with decreasing terminal wind speed. This is consistent with the greater variability of LBVs $\left(\sim 1 \%, v_{\infty} \sim 200 \mathrm{~km} \mathrm{~s}^{-1}\right)$ compared with WRs $(\lesssim 0.2 \%$, $\left.v_{\infty} \gtrsim 1000 \mathrm{~km} \mathrm{~s}^{-1}\right)$.

Investigations into the physical parameters of a single clump required to produce the observed levels of polarization can be found in Nordsieck et al. (2001) and Rodrigues \& Magalhães (2000). The solid angle subtended by the clump must be large enough to scatter a significant fraction of the starlight. Nordsieck et al. (2001) find that the clump must be within $\sim 2.5 R_{\text {star }}$ and be $\sim 20$ times as dense as the ambient wind; while Rodrigues \& Magalhães (2000) show that the clump must be a significant fraction of the size of the star $\left(R_{\text {clump }} \gtrsim 0.5 R_{\text {star }}\right)$. Modelling the observed polarisation variablility of an LBV wind with multiple clumps at various distances in the wind and under a range of geometric distributions (inclination and opening angle) would yield valuable information about the overall wind geometry, as well as the clump production rate.

\subsubsection{Binarity}

If the variability of the intrinsic polarization were to be periodic, this would suggest that the polarimetric variations were due to interactions between the LBV and a binary companion, à la close WR-O systems. Here, the polarization is produced by the electrons in the WR star's fast, ionised wind scattering the light from the bright companion $\mathrm{O}$ star in the region between the two. The polarization describes a double loop in $Q-U$ space about a central locus for each orbital cycle (see St.-Louis et al. 1987). The stars must be sufficiently close together in order for the WR's wind to intercept enough of the O star's light, and consequently the systems have periods of a few days to months. The temporal resolution of present polarimetric observations is insufficient to detect such oscillations in the LBVs' polarization, and so this scenario warrants further invesigation. If a similar mechanism is responsible for the LBV's polarization, the LBV would either have to play the role of the scatterer (i.e. be in a system with a hot star) or the illuminator (i.e. have a WR companion).

If the LBVs were in a system with a WR star, we may expect to see i) X-rays from the collision between the fast WR wind and the LBV wind, cf. the WC8 - evolved O star system HD 68273 (Schild et al. 2004); and ii) other periodic phenomena such as spectroscopic oscillations or the cyclical photometric variability due to the comparable mass of the companion. We note that of the four polarimetrically variable LBVs, only P Cyg has been detected as an X-ray source (Berghöfer \& Wendker 2000), and this has been rated as questionable by the authors. These stars have been subject to timeresolved photometric and spectroscopic monitoring, but while oscillations have been detected, these are on characteristic timescales rather than strictly periodic, and are explained as stellar pulsations (see e.g. Stahl et al. 2001; Lamers et al. 1998).

If the LBV's wind was electron-scattering the light of a companion, the luminosity of the companion would have to be comparable to that of the LBV in order for the scattered light to make up the $\sim 1 \%$ polarization of the overall light we observe (the scattering of an O star's light in WR-O binaries results in typically $\Delta P \sim 0.4 \%$ ). It is unlikely that in this case the companion would not have been already detected.

As an aside, it has been shown that after the periodic components have been subtracted, the polarimetric variations in WR-O binary systems are no greater than for single WR stars (Robert et al. 1989). As these polarimetric variations are associated with wind-clumping in WR stars, this result implies that binarity does not play a significant role in the clumping of hot star winds. In short, binarity is an unlikely explanation for the observed polarimetric variability, either as a scattering mechanism or as a catalyst for wind-clumping.

\section{Conclusions}

From our observations, it is clear that LBVs show a high rate of wind-asphericity. We find evidence for intrinsic polarization in $\gtrsim 50 \%$ of those stars studied, compared with $25 \%$ and $20 \%$ for similar studies of $\mathrm{O}$ supergiants and Wolf-Rayet stars respectively. However, multiple observations reveal levels and angles of intrinsic polarization that are seemingly random with time. In investigating four possible explanations for this we argue the evidence points away from the simple explanation of equatorially-enhanced or bi-polar flows. Instead, we interpret the variable intrinsic polarization as evidence for significant clumping within the wind. The random levels of polarization measured in $\mathrm{R} 127$ are confined to position angles (PAs) of $\pm 10^{\circ}$, which can be explained as an equatorially enhanced, clumpy wind viewed edge-on. If viewed at lower inclination angles, the measured PAs may seem as random as those observed in AG Car, HR Car and P Cyg at limited temporal sampling. Therefore, time-resolved spectropolarimetric monitoring of LBVs would be required in order to detect axi-symmetry in their winds.

We find that wind asphericity is more likely to be found in stars with strong $\mathrm{H} \alpha$ emission and strong recent variability ( $\gtrsim 1 \mathrm{mag}$ ). As both these properties are linked to the stars' massloss rates they may also be linked to the clumping of the wind. With higher mass-loss rates, slower winds and lower effective gravities than their evolutionary neighbours, as well as residing in the bi-stability temperature regime, small changes in radius and/or effective temperature may lead to significant wind instabilities and inhomogeneities. This is consistent with the lower incidence of intrinsic polarization found in $\mathrm{O}$ supergiants and Wolf-Rayet stars.

Acknowledgements. We thank the anonymous referee and Norbert Langer for their helpful comments and suggestions. We wish to thank the staff at the AAT and WHT for their assistance during the observing runs. This work has made extensive use of the online database of SIMBAD and the Starlink software suite, in particular the packages FIGARO, TSP and POLMAP. We have made use of data from the AAT archive and the $\eta$ Car HST Treasury Project. We acknowledge with thanks the variable star observations from the AAVSO International Database contributed by observers worldwide and used in this research. BD and JSV are funded by PPARC. 


\section{Appendix A: Atlas of early-type supergiants}

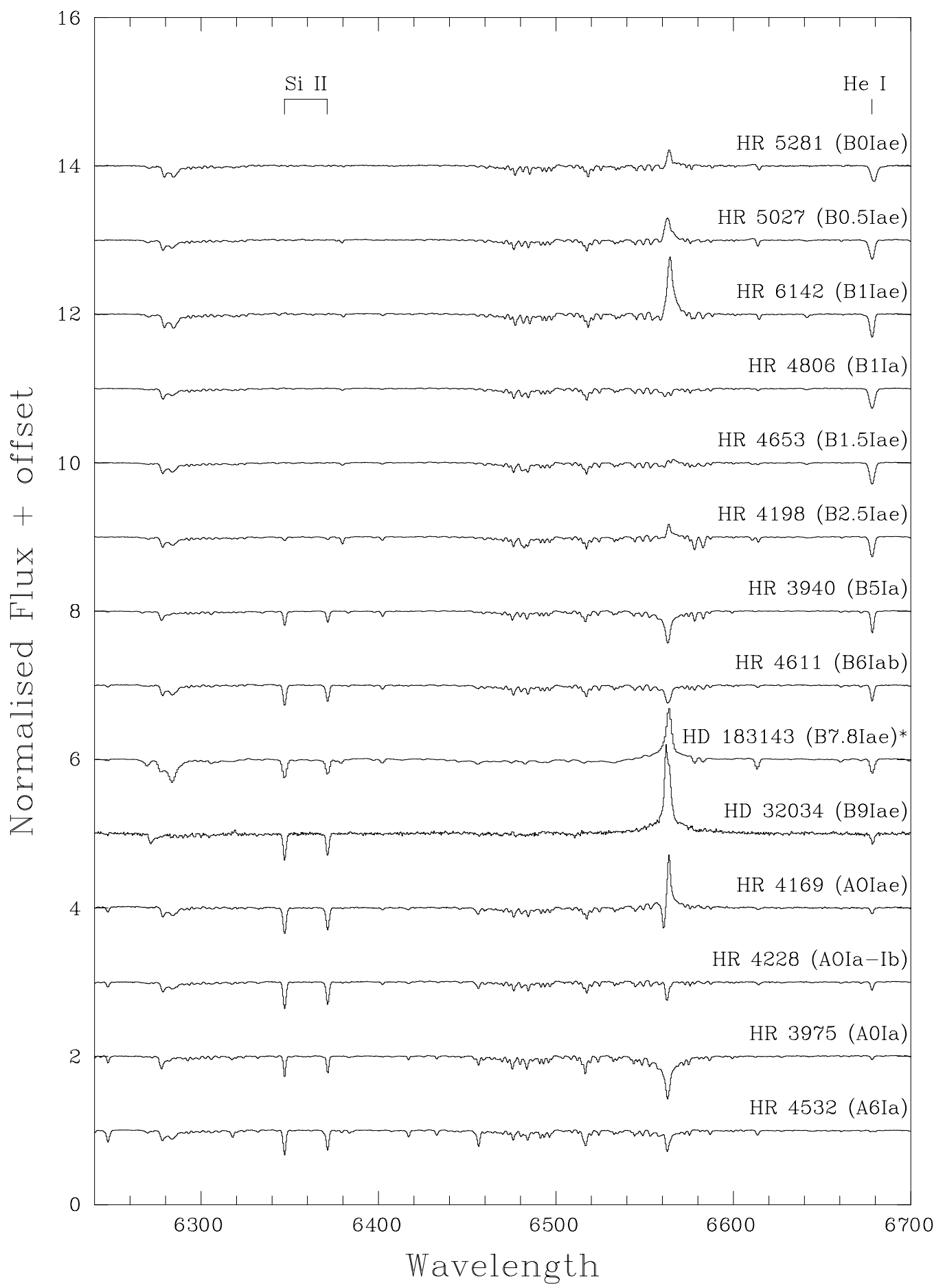

Fig. A.1. A spectral atlas of early-type supergiants in the range 6240-6700 ̊. The prominent photospheric absorption lines of SiII and HeI, which appear to be good indicators of effective stellar temperature, have been identified; and the spectra have been shifted such that these lines are at their rest wavelength. Spectral types of the stars were obtained from SIMBAD. The data in the atlas is available in electronic form at the CDS.

*HD 183143 is a candidate LBV.

\section{References}

Aitken, D. K., \& Hough, J. H. 2001, PASP, 113, 1300

Angel, J. R. P. 1969, ApJ, 158, 219
Barlow, M. J., Drew, J. E., Meaburn, J., \& Massey, R. M. 1994, MNRAS, 268, L29

Berghöfer, T. W., \& Wendker, H. J. 2000, Astron. Nachr., 321, 249 
Cassinelli, J. P., Nordsieck, K. H., \& Murison, M. A. 1987, ApJ, 317, 290

Chesneau, O., Roche, M., Boccaletti, A., et al. 2000, A\&AS, 144, 523

Clampin, M., Nota, A., Golimowski, D. A., Leitherer, C., \& Durrance, S. T. 1993, ApJ, 410, 35

Clampin, M., Schulte-Ladbeck, R. E., Nota, A., et al. 1995, AJ, 110, 251

Cox, P., Mezger, P. G., Sievers, A., et al. 1995, A\&A, 297, 168

Damineli, A., Kaufer, A., Wolf, B., et al. 2000, ApJ, 528, 101

Davidson, K., \& Humphreys, R. M. 1997, ARA\&A, 35, 1

Davidson, K., Humphreys, R. M., Hajian, A., \& Terzian, Y. 1993, ApJ, 411, 336

de Groot, M., \& Lamers, H. J. G. L. M. 1992, Lect. Notes Phys. (Berlin: Springer Verlag), 401, 121

de Koter, A., Lamers, H. J. G. L. M., \& Schmutz, W. 1996, A\&A, 306, 501

Duncan, R. A., \& White, S. M. 2002, MNRAS, 330, 63

Dwarkadas, V. V., \& Balick, B. 1998, AJ, 116, 829

Dwarkadas, V. V., \& Owocki, S. P. 2002, ApJ, 581, 1337

Exter, K. M., Watson, S. K., Barlow, M. J., \& Davis, R. J. 2002, MNRAS, 333, 715

Frank, A., Balick, B., \& Davidson, K. 1995, ApJ, 441, L77

Harries, T. J., \& Howarth, I. D. 1996, A\&A, 310, 533

Harries, T. J., Hillier, D. J., \& Howarth, I. D. 1998, MNRAS, 296, 1072

Harries, T. J., Howarth, I. D., \& Evans, C. J. 2002, MNRAS, 337, 341

Hsu, J.-C., \& Breger, M. 1982, ApJ, 262, 732

Hu, J. Y., de Winter, D., Thé, P. S., \& Perez, M. R. 1990, A\&A, 227, 17

Humphreys, R. M., \& Davidson, K. 1994, PASP, 106, 1025

Humphreys, R. M., Davidson, K., \& Smith, N. 1999, PASP, 111, 1124

Hutsemekers, D. 1997, in Luminous Blue Variables: Massive Stars in Transition, ed. A. Nota, \& H. Lamers, ASP Conf. Ser., 120

Ishibashi, K., Gull, T. R., Davidson, K., et al. 2003, AJ, 125, 3222

Israelian, G., \& de Groot, M. 1999, Space Sci. Rev., 90, 493

Lamers, H. J. G., \& Pauldrach, A. W. A. 1991, A\&A, 244, L5

Lamers, H. J. G. L. M., \& Cassinelli, J. P. 1999, Introduction to Stellar Winds (Cambridge)

Lamers, H. J. G. L. M., Bastiaanse, M. V., Aerts, C., \& Spoon, H. W. W. 1998, A\&A, 335, 605

Lamers, H. J. G. L. M., Snow, T. P., \& Lindholm, D. M. 1995, ApJ, 455,269

Langer, N. 1997, in Luminous Blue Variables: Massive Stars in Transition, ed. A. Nota, \& H. J. G. L. M. Lamers, ASP Conf. Ser., 120,83

Langer, N., García-Segura, G., \& Mac Low, M. 1999, ApJ, 520, L49

Leitherer, C., Allen, R., Altner, B., et al. 1994, ApJ, 428, 292

Maeder, A., \& Meynet, G. 2000, A\&A, 361, 159

Massey, P. 2000, PASP, 112, 144

McLean, I. S. 1979, MNRAS, 186, 265

Meaburn, J., Lopez, J. A., Barlow, M. J., \& Drew, J. E. 1996, MNRAS, 283, L69

Meaburn, J., O'connor, J. A., López, J. A., et al. 2000, MNRAS, 318, 561

Morris, P. W., Waters, L. B. F. M., Barlow, M. J., et al. 1999, Nature, 402, 502

Morse, J. A., Davidson, K., Bally, J., et al. 1998, AJ, 116, 2443

Nordsieck, K. H., Wisniewski, J., Babler, B. L., et al. 2001, in P Cygni 2000: 400 Years of Progress, ed. M. de Groot, \& C. Sterken, ASP Conf. Ser., 233

Nota, A. \& Clampin, M. 1997, in Luminous Blue Variables: Massive Stars in Transition, ed. A. Nota, \& H. Lamers, ASP Conf. Ser., 120
Nota, A., Leitherer, C., Clampin, M., Greenfield, P., \& Golimowski, D. A. 1992, ApJ, 398, 621

Nota, A., Livio, M., Clampin, M., \& Schulte-Ladbeck, R. 1995, ApJ, 448,788

Nota, A., Smith, L., Pasquali, A., Clampin, M., \& Stroud, M. 1997, ApJ, 486, 338

Oudmaijer, R. D., \& Drew, J. E. 1999, MNRAS, 305, 166

Oudmaijer, R. D., Proga, D., Drew, J. E., \& de Winter, D. 1998, MNRAS, 300, 170

Parthasarathy, M., Jain, S. K., \& Bhatt, H. C. 2000, A\&A, 355, 221

Pelupessy, I., Lamers, H. J. G. L. M., \& Vink, J. S. 2000, A\&A, 359, 695

Robert, C., Moffat, A. F. J., Bastien, P., Drissen, L., \& St.-Louis, N. 1989, ApJ, 347, 1034

Rodrigues, C. V., \& Magalhães, A. M. 2000, ApJ, 540, 412

Schild, H., Güdel, M., Mewe, R., et al. 2004, A\&A, 422, 177

Schmidt-Kaler, T. 1982, Landolt-Bornstein Gp. 6, Vol. 2-2b (Berlin: Springer-Verlag)

Schulte-Ladbeck, R. E., Clayton, G. C., Hillier, D. J., Harries, T. J., \& Howarth, I. D. 1994, ApJ, 429, 846

Schulte-Ladbeck, R. E., Leitherer, C., Clayton, G. C., et al. 1993, ApJ, 407, 723

Schulte-Ladbeck, R. E., Pasquali, A., Clampin, M., et al. 1999, AJ, 118,1320

Serkowski, K., Mathewson, D. L., \& Ford, V. L. 1975, ApJ, 196, 261

Smith, L. J., Nota, A., Pasquali, A., et al. 1998, ApJ, 503, 278

Smith, N., Davidson, K., Gull, T. R., Ishibashi, K., \& Hillier, D. J. 2003a, ApJ, 586, 432

Smith, N., Gehrz, R. D., Hinz, P. M., et al. 2003b, AJ, 125, 1458

Smith, N., Vink, J. S., \& de Koter, A. 2004, ApJ, 615, 475

St.-Louis, N., Drissen, L., Moffat, A. F. J., Bastien, P., \& Tapia, S. 1987, ApJ, 322, 870

Stahl, O. 1987, A\&A, 182, 229

Stahl, O., Gäng, T., Sterken, C., et al. 2003, A\&A, 400, 279

Stahl, O., Jankovics, I., Kovács, J., et al. 2001, A\&A, 375, 54

Stahl, O., Wolf, B., Klare, G., et al. 1983, A\&A, 127, 49

Sterken, C., de Groot, M., \& van Genderen, A. M. 1998, A\&A, 333, 565

Sterken, C., Gosset, E., Juttner, A., et al. 1991, A\&A, 247, 383

Taylor, M., Nordsieck, K. H., Schulte-Ladbeck, R. E., \& Bjorkman, K. S. 1991, AJ, 102, 1197

van Boekel, R., Kervella, P., Schöller, M., et al. 2003, A\&A, 410, 37

van Genderen, A. M. 2001, A\&A, 366, 508

van Genderen, A. M., \& Sterken, C. 1999, A\&A, 349, 537

van Genderen, A. M., Thé, P. S., de Winter, D., et al. 1992, A\&A, 258, 316

van Genderen, A. M., de Groot, M., \& Sterken, C. 1997, A\&AS, 124, 517

Vink, J. S., \& de Koter, A. 2002, A\&A, 393, 543

Vink, J. S., de Koter, A., \& Lamers, H. J. G. L. M. 1999, A\&A, 350, 181

Vink, J. S., Drew, J. E., Harries, T. J., \& Oudmaijer, R. D. 2002, MNRAS, 337, 356

Vink, J. S., Drew, J. E., Harries, T. J., Oudmaijer, R. D., \& Unruh, Y. C. 2003, A\&A, 403, 703

Voors, R. H. M., Waters, L. B. F. M., Morris, P. W., et al. 1999, A\&A, 341,67

Waagen, E. O. 2005, private communication

Weis, K. 2003, A\&A, 408, 205

Wolf, B., Campusano, L., \& Sterken, C. 1974, A\&A, 36, 87

Wood, K., Bjorkman, J. E., Whitney, B. A., \& Code, A. D. 1996, ApJ, 461,828 